\title{
A General Framework for Performance Analysis of Space Shift Keying (SSK) Modulation for MISO Correlated Nakagami- $m$ Fading Channels
}

\author{
Marco Di Renzo, Member, IEEE, and Harald Haas, Member, IEEE
}

\begin{abstract}
In this paper, we offer an accurate framework for analyzing the performance of wireless communication systems adopting the recently proposed Space Shift Keying (SSK) modulation scheme. More specifically, we study the performance of a $N_{t} \times 1$ MISO (Multiple-Input-Single-Output) system setup with Maximum-Likelihood (ML) detection and full Channel State Information (CSI) at the receiver. The exact Average Bit Error Probability (ABEP) over generically correlated and nonidentically distributed Nakagami- $m$ fading channels is computed in closed-form when $N_{t}=2$, while very accurate and asymptotically tight upper bounds are proposed to compute the ABEP when $N_{t}>2$. With respect to current literature, our contribution is threefold: $i)$ the ABEP is computed in closed-form without resorting to Monte Carlo numerical simulations, which, besides being computationally intensive, only yield limited insights about the system performance and cannot be exploited for a systematic optimization of it, ii) the framework accounts for arbitrary fading conditions and is not restricted to identically distributed fading channels, thus offering a comprehensive understanding of the performance of SSK modulation over generalized fading channels, and iii) the analytical framework could be readily adapted to study the performance over generalized fading channels with arbitrary fading distributions, since the Nakagami$m$ distribution is a very flexible fading model, which either includes or can closely approximate several other fading models. Numerical results show that the performance of SSK modulation is significantly affected by the characteristics of fading channels, e.g., channel correlation, fading severity, and power imbalance among the $N_{t}$ transmit-receive wireless links. Analytical frameworks and theoretical findings are also substantiated via Monte Carlo simulations.
\end{abstract}

Index Terms-Spatial modulation (SM), space shift keying (SSK) modulation, multiple-input-single-output (MISO) systems, performance analysis, correlated fading channels, Nakagami- $m$ fading.

Paper approved by G. Bauch, the Editor for MIMO, Coding and Relaying of the IEEE Communications Society. Manuscript received September 17, 2009; revised January 18, 2010.

This paper was presented in part at the IEEE International Conference on Communications (ICC), Cape Town, South Africa, May 2010.

M. Di Renzo is with L2S, UMR 8506 CNRS-SUPELEC-Univ Paris-Sud, 3 rue Joliot-Curie, 91192 Gif-sur-Yvette CEDEX (Paris), France (e-mail: marco.direnzo@1ss.supelec.fr).

H. Haas is with The University of Edinburgh, College of Science and Engineering, School of Engineering, Institute for Digital Communications (IDCOM), Alexander Graham Bell Building, King's Buildings, Mayfield Road, Edinburgh, EH9 3JL, Scotland, United Kingdom (UK) (e-mail: h.haas@ed.ac.uk).

Digital Object Identifier 10.1109/TCOMM.2010.09.090565

\section{INTRODUCTION}

$\mathbf{T}$ HE use of multiple antennas for wireless communication systems has received an upsurge of research interest during the last decade, both in academia and industry [1]. Among the many transmitter and receiver concepts proposed so far, Spatial Modulation (SM) is a novel and recently proposed wireless transmission technique for Multiple-InputMultiple-Output (MIMO) wireless systems [2], [3]. Recent research studies have pointed out that it can be a promising candidate to the design of low-complexity modulation schemes and transceiver architectures for MIMO systems over fading channels [4]-[6].

In particular, it has been shown that SM can offer, without reducing the achievable data rate, better performance than other popular MIMO communication systems, e.g., V-BLAST (Vertical Bell Laboratories Layered Space-Time) [7], Alamouti [8], and Amplitude Phase Modulation (APM) schemes [4]-[6]. Furthermore, these performance gains are obtained with a significant reduction in receiver complexity and system design: SM can efficiently avoid Inter-Channel Interference (ICI) and Inter-Antenna Synchronization (IAS) issues of conventional MIMO systems, as well as reduce the number of computations required by the detection unit [4], [5]. Moreover, only one RF (Radio Frequency) front-end chain is required, in theory, at the transmitter-side [6], which significantly reduces the overall complexity of the system. More specifically, lowcomplexity transceiver designs and high spectral efficiencies are simultaneously achieved by adopting a simple modulation and coding mechanism that foresees to activate a single transmit-antenna at any time instant and to use its spatial position as an additional dimension for transmitting information.

\section{A. Historical Perspective and Terminology}

The fundamental and distinguishable feature that makes SM different from other MIMO techniques is the exploitation, as a source of information, of the spatial constellation pattern (i.e., the spatial position in the antenna-array) of the transmitantennas. Along the recent history, this "space modulation" concept has been introduced for different application scenarios and has been termed in different ways with slightly different meanings. The first published contribution on the matter is [2], where the method has been called Space Shift Keying (SSK) modulation. In [2], the idea of exploiting the differences in the signals received from different antennas to discriminate the transmitted information messages has been described for the 
first time. In particular, two approaches have been introduced in [2]: i) a first one with reduced data rate and receiver complexity, where the information to be sent is encoded only on the spatial positions of the transmit-antennas, and ii) a second one with higher date rate and receiver complexity, where the information bits are encoded on both spatial and signal constellation diagrams. However, the idea in [2] is useful for a simple $2 \times 1$ MIMO system, still requires IAS and multiple RF chains, and suffers from ICI. One year later, in [3] the principle of using the positions of the transmit-antennas as a source of information has been explicitly exploited, for the first time, to accomplish a form of multiplexing in the spatial domain rather than, as usually done in Orthogonal Frequency-Division Multiplexing (OFDM) systems, in the frequency domain. For this reason the method has been called Orthogonal Spatial-Division Multiplexing (OSDM). OSDM fully avoids ICI, does not require IAS, and only needs one RF chain, at the expense of some loss in spectral efficiency due to additional parity bits. In [4] and [6] the ideas in [2] and [3] have been further elaborated to conceive two new modulation schemes that can be used for MIMO systems with arbitrary transmit- and receive-antennas, and that completely avoid ICI, IAS, multiple RF chains, as well as the spectral efficiency loss due to the parity bits in [3]. In particular, the schemes in [4] and [6] can be related to the second and first approach proposed in [2], respectively. However, they show a fundamental difference with respect to [2]: in [2], multiple transmit-antennas can be activated at any time instant, while in [4] and [6] only one transmit-antenna can radiate power at any time instant. The MIMO scheme in [4] has been called SM, while in [6] the authors have retained the name SSK modulation. Broadly speaking, the method proposed in [6] is a special instance of the SM scheme introduced in [4], which has reduced receiver complexity but lower data rate. Throughout this paper, we adopt the term SM to refer to the generic concept of "space modulation" described in [4], while we use the term SSK modulation to refer to the special case of SM described in [6], where the information bits are encoded only on the spatial positions of the transmit-antennas.

\section{B. State of the Art and Limitations}

The underlying principle of SM is twofold [4]: i) at the transmitter, a one-to-one mapping of information bits into two information carrying units, i.e., a symbol that is chosen from a signal-constellation diagram, and a unique transmit-antenna index that is chosen from the set of transmit-antennas in the antenna-array, and ii) at the receiver, the exploitation, due to the properties of wireless fading channels, of distinct multipath profiles along any available transmit-receive wireless links [9]. Moving from this basic working principle, the following research contributions on SM are available in the literature to date. i) In [4], a heuristic receiver design for SM is proposed, and its performance analyzed over correlated fading channels. ii) In [5], the optimal detector for SM with full Channel State Information (CSI) at the receiver is developed, and its performance studied over uncorrelated Rayleigh fading channels. iii) In [6], the optimal detector for SSK modulation is introduced, and its performance analyzed for uncoded and coded systems. iv) In [10], an optimization framework to allow more than one transmit-antenna at a time to convey information is introduced, and some performance improvements, due to the optimal constellation design, are shown.

Numerical studies in, e.g., [4], [6], have pointed out that a fundamental issue to be taken into account for the accurate analysis, design, and optimization of SM is channel correlation among the signals received by the transmit-antennas. As a matter of fact, at the receiver-side, the optimal detector [5] is designed to exploit the distinct multipath profiles of different wireless links: if correlation exist among them, it may be unable to distinguish the different antennas, which will appear almost the same at the receiver. In order to cope with channel correlation, a novel scheme named Trellis Coded Spatial Modulation (TCSM) is introduced in [11], which exploits trellis coding to reduce, on average, the effect of fading spatial correlation. However, all performance evaluations conducted in correlated fading channels for SM are based on Monte Carlo numerical simulations to date, which, besides being computationally intensive, only yield limited insights about the system performance and cannot be exploited for a systematic optimization of it. To the best of the authors knowledge, only in [12] the authors have attempted to develop a framework to analyze the performance of SM over correlated Nakagami- $m$ fading channels. However, this latter framework shows three main limitations: i) the detector is suboptimal, ii) the proposed method is semi-analytical, and, more important, iii) fading correlation is taken into account only for data detection, while the probability of transmit-antenna detection is computed by using the framework in [4], which neglects fading correlation. In addition to all the above, the analysis in [4]-[6] is implicitly restricted to identically distributed fading channels, thus not offering a comprehensive understanding of the performance of SM over generalized fading channels [13].

\section{Contribution}

Motivated by the lack of general communication-theoretic frameworks for the analysis and design of SM over correlated fading channels, in this paper we propose an accurate analytical framework to study the performance of SSK modulation over Nakagami- $m$ fading channels, by explicitly taking into account spatial correlation effects and arbitrary fading parameters among any transmit-receive path. More specifically, we will focus our attention on the $N_{t} \times 1$ MISO (MultipleInput-Single-Output) system setup $\left(N_{t}\right.$ denotes the number of antennas at the transmitter) with Maximum-Likelihood (ML) detection and full CSI at the receiver. By exploiting the Moment Generating Function (MGF-) based approach for performance analysis of digital communication systems over fading channels [13], single-integral closed-form expressions for the Average Bit Error Probability (ABEP) will be provided.

More specifically, the novelties and contributions of the paper are as follows. i) Differently from other results available to date (see, e.g., [4]-[6], [12]), the ABEP over correlated fading channels is computed analytically and in closed-form. ii) Exact frameworks and asymptotically tight upper bounds are developed for $N_{t}=2$ and $N_{t}>2$, respectively. In particular, when $N_{t}>2$ we show that, differently from 
[6], our bounds do no require any ad-hoc correction term to be very tight. iii) We consider very general correlation models and arbitrary fading parameters in all wireless links. iv) We point out that the performance of SSK modulation is significantly affected by different channel fading conditions, and, particularly, besides channel correlation, by the power imbalance among the transmit-receive wireless paths. To the best of the authors knowledge, this latter aspect has never been studied before in the literature. v) With respect to the vast majority of communication-theoretic frameworks available in the literature [13], we point out that the ABEP of SSK modulation depends on the complex channel gains of the fading channel rather than just on the channel envelopes. To account for this peculiarity, a completely novel communication-theoretic framework for performance analysis will be proposed. Finally, we note that although several others fading models exist in the literature [13], [14], it is well-known that the Nakagami$m$ distribution is a very flexible fading model, which either includes or can closely approximate several other fading distributions [13]. Moreover, it has been recently shown in [15], [16] that it represents the building block for the accurate performance analysis of wireless systems over generalized fading channels with arbitrary fading distributions. Accordingly, the results in this paper can be readily extended to other fading models by using [13], [15], and [16]. On the contrary, the generalization of the framework from SSK modulation to SM is left to a future research contribution, due to the analytical complexity of the problem.

\section{Paper Organization}

The remainder of the manuscript is organized as follows. In Section II, system, channel models, and optimal ML detector are introduced. In Section III, the analytical framework for performance analysis of $2 \times 1$ MISO systems over independent and generically correlated Nakagami- $m$ fading channels is developed. In Section IV, the framework in Section III is extended to multiple $\left(N_{t}>2\right)$ transmit-antennas by capitalizing on some tight upper bounds. In Section V, numerical and simulation results are shown to substantiate the accuracy of the analytical framework. Finally, Section VI concludes the paper.

\section{SySTEM MOdEL AND BACKGROUND}

Let us consider a generic $N_{t} \times 1$ MISO system, with $N_{t}$ being the number of antennas at the transmitter. As mentioned in Section I, SSK modulation foresees: i) at the transmitter, to map information data bits to transmit-antennas indexes only, and ii) at the receiver, to de-map these bits via suitable detection mechanisms to estimate, for each signaling timeinterval, the active transmit-antenna. Data transmission is based on the following fundamental principles [6]: i) activate the transmit-antenna which is linked to the sequence of bits to be transmitted, and ii) switch off the rest of the transmitantennas. This way, the estimation, at the receiver-side, of which antenna is not idle results, implicity, in the estimation of the unique sequence of bits emitted by the encoder at the transmitter-side. The detection mechanism of the antenna index is based upon the location-specific and distinct fingerprint of the wireless channel along any transmit-receive path [9]. In particular, the detection process at the receiver can be cast in terms of a general $N_{t}$-hypothesis detection problem in Additive White Gaussian Noise (AWGN) [17, Sec. 4.2, pp. 257], when conditioning upon fading channel statistics. In Section II-C, we will briefly summarize the detection problem for SSK modulation, as well as describe the optimal ML detector when full CSI at the receiver is available [6].

\section{A. Notation}

Let us briefly introduce the main notation used in what follows. i) We adopt a complex-envelope signal representation. ii) $j=\sqrt{-1}$ is the imaginary unit. iii) $(\cdot)^{*}$ denotes complexconjugate. iv) $(x \otimes y)(t)=\int_{-\infty}^{+\infty} x(\xi) y(t-\xi) d \xi$ is the convolution of signals $x(\cdot)$ and $y(\cdot)$. v) $|\cdot|^{2}$ denotes square absolute value. vi) $\mathrm{E}\{\cdot\}$ is the expectation operator. vii) $\operatorname{Re}\{\cdot\}$ denotes the real part operator. viii) $\operatorname{Pr}\{\cdot\}$ means probability. ix) $\rho_{\mathrm{AB}}$ denotes the correlation coefficient of Random Variables (RVs) A and B. x) $Q(x)=(1 / \sqrt{2 \pi}) \int_{x}^{+\infty} \exp \left(-t^{2} / 2\right) d t$ is the Q-function. xi) $\left\{u_{i}\right\}_{i=1}^{N_{t}}$ denotes the set of information messages that the encoder at the transmitter can emit with equal probability. xii) $\hat{u}$ denotes the message estimated at the receiver-side. xiii) $E_{u}$ is the average energy transmitted by each antenna that emits a non-zero signal. xiv) $T_{u}$ denotes the signaling interval for each information message $\left\{u_{i}\right\}_{i=1}^{N_{t}}$. $\mathrm{xv})$ The noise at the receiver input is denoted by $n(\cdot)$, and is assumed to be AWG-distributed, with both real and imaginary parts having a double-sided power spectral density equal to $N_{0}$. xvi) For ease of notation, we set $\bar{\gamma}=E_{u} /\left(4 N_{0}\right)$. xvii) $\left\{s_{n}\left(\cdot \mid\left\{u_{i}\right\}_{i=1}^{N_{t}}\right)\right\}_{n=1}^{N_{t}}$ denotes the signal emitted by the $n$-th transmit-antenna conditioned upon the information message $\left\{u_{i}\right\}_{i=1}^{N_{t}}$. Moreover, $w(\cdot)$ denotes the unit-energy (i.e., $\int_{-\infty}^{+\infty}|w(t)|^{2} d t=1$ ) elementary pulse waveform for each transmission. xviii) $\Gamma(\cdot)$ is the Gamma function [18, Eq. (6.1.1)]. xix) $I_{\nu}(\cdot)$ is the modified Bessel function of first kind and order $\nu\left[18\right.$, Ch. 9)]. xx) $G_{p, q}^{m, n}\left(. \mid \begin{array}{c}\left(a_{p}\right) \\ \left(b_{q}\right)\end{array}\right)$ is the Meijer-G function defined in [19, Ch. 8, pp. 519]. xxi) $\delta(\cdot)$ is the Dirac delta function. xxii) $L_{n}^{\nu}(\cdot)$ is the Generalized Laguerre polynomial in [18, Eq. (22.2.12)]. xxiii) $M_{A}(s)=\mathrm{E}\{\exp (-s A)\}$ is the MGF of RV A. xxiv) $(\cdot)_{n}$ is the Pochhammer symbol, which is defined as $(a)_{n}=$ $\Gamma(a+n) / \Gamma(a) . \operatorname{xxv})($.$) denotes the binomial coefficient.$ xxvi) rect $(\cdot)$ denotes a rectangular pulse that is defined as $\operatorname{rect}\left(\left(t-t_{0}\right) / T_{0}\right)=1$ if $-T_{0} / 2 \leq t-t_{0} \leq T_{0} / 2$ and $\operatorname{rect}\left(\left(t-t_{0}\right) / T_{0}\right)=0$ elsewhere.

\section{B. Channel Model}

We consider a frequency-flat slowly-varying fading channel model, with fading envelopes distributed according to a Nakagami- $m$ distribution ${ }^{1}$ [13]. Moreover, we assume the

\footnotetext{
${ }^{1}$ We emphasize here that for some scenarios of interest a more general frequency-selective fading channel model would be more appropriate. However, at the time of writing, the analysis of the performance of SSK modulation over frequency-selective fading channels seems to be very complicated from the analytical point of view, and, as a consequence, it is considered for a future research contribution.
} 
fading gains not to be necessarily identically distributed, and spatial correlation among them is accounted for in this manuscript. In particular:

- $\left\{h_{i}(t)\right\}_{i=1}^{N_{t}}=\beta_{i} \exp \left(j \varphi_{i}\right) \delta\left(t-\tau_{i}\right)$ is the channel impulse response of the $i$-th wireless link, where $\left\{\beta_{i}\right\}_{i=1}^{N_{t}}$, $\left\{\varphi_{i}\right\}_{i=1}^{N_{t}}$, and $\left\{\tau_{i}\right\}_{i=1}^{N_{t}}$ denote gain, phase, and delay, respectively. Moreover, $\left\{\alpha_{i}\right\}_{i=1}^{N_{t}}=\beta_{i} \exp \left(j \varphi_{i}\right)$ denotes the channel complex gain.

- $\left\{\tau_{i}\right\}_{i=1}^{N_{t}}$ is assumed to be independent and uniformly distributed in $\left[0, T_{u}\right)$, but known at the receiver, i.e., perfect time-synchronization is considered. Similar to [4]-[6], we assume $\tau_{1} \cong \tau_{2} \cong \ldots \cong \tau_{N_{t}}$, which is a realistic hypothesis when the distance between transmitter and receiver is much larger than the spacing between the transmit-antennas ${ }^{2}$, and, to a first-order, the signals transmitted by the antennas differ only in phase [20, Eq. (7.24)]. The assumptions of perfect time-synchronization at the receiver and almost equal propagation delays allow us to neglect $\left\{\tau_{i}\right\}_{i=1}^{N_{t}}$ from our notation and subsequent analysis.

- $\left\{\varphi_{i}\right\}_{i=1}^{N_{t}}$ is assumed to be independent and uniformly distributed in $[0,2 \pi)$.

- The channel envelopes, $\left\{\beta_{i}\right\}_{i=1}^{N_{t}}$, are assumed to be distributed according to a multivariate Nakagami- $m$ distribution. In particular, when $N_{t}=2$ various joint Probability Density Functions (PDFs), $\left\{f_{\beta_{i} \beta_{j}}(\cdot)\right\}_{i \neq j=1}^{N_{t}}$, will be considered in Section III, such as [13, Eq. (6.1)] and [21, Eq. (12)]. In particular, [21, Eq. (12)] is the most general formulation of the PDF of bivariate Nakagami$m$ RVs with arbitrary correlation and fading parameters. By using the tight upper bounds in Section IV, we will see that, to analyze the performance of the $N_{t} \times 1$ MISO system in hand, only the joint PDF of pairs of Nakagami- $m$ RVs is required. This allows us to avoid the complicated expressions for the multivariate Nakagami$m$ distribution (see, e.g., [22], [23]) available in the literature, but still allows us to maintain a very good accuracy of the framework.

- The fading parameters of the $i$-th wireless link are denoted by $\left\{m_{i}\right\}_{i=1}^{N_{t}}$ and $\left\{\Omega_{i}\right\}_{i=1}^{N_{t}}=\mathrm{E}\left\{\beta_{i}^{2}\right\}$.

\section{Optimal ML Detector With Full CSI}

Let $\left\{u_{l}\right\}_{l=1}^{N_{t}}$ be the actual transmitted message. Moving from the above system and channel models, the signals after propagation through the wireless fading channel are $\left\{\tilde{s}_{n}\left(t \mid\left\{u_{l}\right\}_{l=1}^{N_{t}}\right)\right\}_{n=1}^{N_{t}}=\left(s_{n} \otimes h_{n}\right)(t)=$ $\beta_{n} \exp \left(j \varphi_{n}\right) s_{n}\left(t \mid u_{l}\right)$, and the received signal can be written as follows:

$$
r(t)=\bar{s}_{l}(t)+n(t) \quad \text { if } u_{l} \text { is sent }
$$

which is a general $N_{t}$-hypothesis detection problem [13, Sec. 7.1], [17, Sec. 4.2, pp. 257] with $\bar{s}_{l}(t)=\sum_{n=1}^{N_{t}} \tilde{s}_{n}\left(t \mid u_{l}\right)$.

\footnotetext{
${ }^{2}$ We note that this assumption might be avoided when restricting the pulse waveform $w(\cdot)$ to be a pure sine wave, i.e., $w(t)=\operatorname{rect}\left(\left(t-0.5 T_{u}\right) / T_{u}\right)$. In fact, in this case the propagation delays can be embedded, with a slight abuse of notation, into the channel phases and, under the assumptions of this paper, the analysis of the system will be independent of the channel delays. The performance study conducted in this paper applies to this special case as well.
}

Note that (1) is a general hypothesis testing problem where all transmit-antennas could be activated when a message is transmitted [2], [10].

Moving from (1), the optimal ML detector with full CSI and perfect time-synchronization at the receiver is as follows ${ }^{3}$ [5], [13, Sec. 7.1]:

$$
\hat{u}=\underset{\left\{u_{i}\right\}_{i=1}^{N_{t}}}{\arg \max }\left\{D_{i}\right\}
$$

where $\left\{D_{i}\right\}_{i=1}^{N_{t}}$ is the decision metric defined in what follows:

$$
D_{i}=\operatorname{Re}\left\{\int_{T_{u}} r(t) \bar{s}_{i}^{*}(t) d t\right\}-\frac{1}{2} \int_{T_{u}} \bar{s}_{i}(t) \bar{s}_{i}^{*}(t) d t
$$

In particular, (2) tells us that, if the transmitted message is $u_{l}$, the detector will be successful in detecting it, i.e., $\hat{u}=u_{l}$, if and only if $\max _{\left\{u_{i}\right\}_{i=1}^{N_{t}}}\left\{D_{i}\right\}=D_{l}$.

According to [6], SSK modulation overcomes ICI and IAS problems of conventional MIMO systems by allowing only one antenna at the transmitter to be active at any time instant. In formulas, we have:

$$
\bar{s}_{l}(t)=\tilde{s}_{l}\left(t \mid u_{l}\right)
$$

which means that only the $l$-th antenna is activated when $u_{l}$ has to be transmitted.

Moreover, by letting $\left\{s_{l}\left(t \mid u_{l}\right)\right\}_{l=1}^{N_{t}}=\sqrt{E_{u}} w(t)$, (1) simplifies as follows:

$$
r\left(t \mid u_{l}\right)=\sqrt{E_{u}} \beta_{l} \exp \left(j \varphi_{l}\right) w(t)+n(t)
$$

\section{ABEP OVER CORRELATED NAKAGAMI- $m$ FAding Channels: The $2 \times 1$ MISO SETUP}

Let us consider $N_{t}=2$. From the decision rule in (2), the probability of error, $\mathrm{P}_{\mathrm{E}}(\cdot, \cdot)$, of the detection process (i.e., the detection of the index of the transmit-antenna), when conditioning upon the channel impulse responses $\left\{h_{i}(\cdot)\right\}_{i=1}^{2}$, can be explicitly written as follows:

$$
\begin{aligned}
& \mathrm{P}_{\mathrm{E}}\left(h_{1}, h_{2}\right)=\left.\frac{1}{2} \mathrm{P}_{\mathrm{E}}\left(h_{1}, h_{2}\right)\right|_{u_{1}}+\left.\frac{1}{2} \mathrm{P}_{\mathrm{E}}\left(h_{1}, h_{2}\right)\right|_{u_{2}} \\
& =\frac{1}{2} \operatorname{Pr}\left\{\left.D_{1}\right|_{u_{1}}<\left.D_{2}\right|_{u_{1}}\right\}+\frac{1}{2} \operatorname{Pr}\left\{\left.D_{2}\right|_{u_{2}}<\left.D_{1}\right|_{u_{2}}\right\}
\end{aligned}
$$

where $\left\{\left.\mathrm{P}_{\mathrm{E}}(\cdot, \cdot)\right|_{u_{l}}\right\}_{l=1}^{2}$ and $\left\{\left.D_{i}\right|_{u_{l}}\right\}_{i, l=1}^{2}$ denote the probabilities of error and the decision metrics conditioned upon the transmission of messages $\left\{u_{l}\right\}_{l=1}^{2}$, respectively.

\section{A. ABEP: Preliminaries}

1) Conditional BEP - Fixed Channel Realization: By assuming the channel coefficients to be known and fixed, the decision metrics $\left\{\left.D_{i}\right|_{u_{l}}\right\}_{i, l=1}^{2}$ can be obtained by substituting (5) into (3), as shown in what follows:

$$
\left\{\begin{array}{l}
\left\{\left.D_{l}\right|_{u_{l}}\right\}_{l=1}^{2}=\frac{1}{2} \beta_{l}^{2} E_{u}+\beta_{l} \sqrt{E_{u}} \tilde{n}_{l} \\
\left\{\left.D_{i}\right|_{u_{l}}\right\}_{i \neq l=1}^{2}=\operatorname{Re}\left\{\alpha_{l} \alpha_{i}^{*}\right\} E_{u}-\frac{1}{2} \beta_{i}^{2} E_{u}+\beta_{i} \sqrt{E_{u}} \tilde{n}_{i}
\end{array}\right.
$$

${ }^{3}$ In order to avoid any confusion with the adopted notation, let us emphasize that the subscript $l$ denotes the actual message that is transmitted, while the subscript $i$ denotes the (generic) $i$-th message that is tested by the detector to solve the $N_{t}$-hypothesis detection problem. More specifically, for each signaling interval, $l$ is fixed, while $i$ can take different values at the detector. 
with $\left\{\tilde{n}_{i}\right\}_{i=1}^{2}=\operatorname{Re}\left\{\int_{T_{u}} n(t) \exp \left(-j \varphi_{i}\right) w^{*}(t) d t\right\}$.

From (7), $\left.\mathrm{P}_{\mathrm{E}}(\cdot, \cdot)\right|_{u_{1}}$ can be written, after a few algebraic manipulations, as follows:

$$
\left.\mathrm{P}_{\mathrm{E}}\left(h_{1}, h_{2}\right)\right|_{u_{1}}=\operatorname{Pr}\left\{\left|\alpha_{2}-\alpha_{1}\right|^{2} E_{u}<\tilde{n}\right\}
$$

where we have defined $\tilde{n}=2 \beta_{2} \sqrt{E_{u}} \tilde{n}_{2}-2 \beta_{1} \sqrt{E_{u}} \tilde{n}_{1}$, which, when conditioning upon the fading channel gains, is a Gaussian-distributed RV with zero-mean and variance $\mathrm{E}\left\{\tilde{n}^{2}\right\}=4 N_{0} E_{u}\left|\alpha_{2}-\alpha_{1}\right|^{2}$.

Accordingly, $\left.\mathrm{P}_{\mathrm{E}}(\cdot, \cdot)\right|_{u_{1}}$ can be readily computed in closed-form as follows [17, Sec. 2.2.2]:

$$
\left.\mathrm{P}_{\mathrm{E}}\left(h_{1}, h_{2}\right)\right|_{u_{1}}=Q\left(\sqrt{\bar{\gamma}\left|\alpha_{2}-\alpha_{1}\right|^{2}}\right)
$$

By using similar analytical steps, it can be shown that $\left.\mathrm{P}_{\mathrm{E}}\left(h_{1}, h_{2}\right)\right|_{u_{2}}=Q\left(\sqrt{\bar{\gamma}\left|\alpha_{2}-\alpha_{1}\right|^{2}}\right)$, from which $\mathrm{P}_{\mathrm{E}}(\cdot, \cdot)$ in (6) becomes:

$$
\mathrm{P}_{\mathrm{E}}\left(h_{1}, h_{2}\right)=Q\left(\sqrt{\bar{\gamma}\left|\alpha_{2}-\alpha_{1}\right|^{2}}\right)
$$

We observe that the final expression of the conditional BEP is very different from other multiple-antenna techniques. For instance, it is different from typical Equal Gain Combining (EGC) and Maximal Ratio Combining (MRC) schemes (see, e.g., [13], [24], [25]). In particular, there are two main differences: i) $\mathrm{P}_{\mathrm{E}}(\cdot, \cdot)$ depends on the difference between the complex channel gains, which is a clear indication that SSK modulation can exploit only in part the multiple antennas at the transmitter for diversity purposes [6], [26], and ii) besides the fading envelopes $\left\{\beta_{i}\right\}_{i=1}^{2}, \mathrm{P}_{\mathrm{E}}(\cdot, \cdot)$ is strongly affected by the channel phases $\left\{\varphi_{i}\right\}_{i=1}^{2}$ as well. As a consequence, novel specific communication-theoretic frameworks seem to be required to understand and analyze the performance of SSK modulation over fading channels. In what follows, some novel frameworks to compute the ABEP will be introduced by exploiting the MGF-based approach for performance analysis of digital communication systems over fading channels [13].

2) MGF-based Approach: By exploiting the MGF-based approach, the $\mathrm{ABEP}$, i.e., $\mathrm{ABEP}=\mathrm{E}\left\{\mathrm{P}_{\mathrm{E}}\left(h_{1}, h_{2}\right)\right\}$, can be obtained from [13, Eq. (5.1), Eq. (5.3)], as shown in what follows:

$$
\mathrm{ABEP}=\mathrm{E}\left\{\mathrm{P}_{\mathrm{E}}\left(h_{1}, h_{2}\right)\right\}=\frac{1}{\pi} \int_{0}^{\pi / 2} M_{\gamma}\left(\frac{\bar{\gamma}}{2 \sin ^{2}(\theta)}\right) d \theta
$$

where $M_{\gamma}(\cdot)$ is the MGF of RV $\gamma$, and $\gamma=\left|\alpha_{2}-\alpha_{1}\right|^{2}=$ $\left|\beta_{2} \exp \left(j \varphi_{2}\right)-\beta_{1} \exp \left(j \varphi_{1}\right)\right|^{2}$.

The MGF, $M_{\gamma}(\cdot)$, of $\gamma$, can be explicitly written as a function of the joint PDF $f_{\beta_{1} \beta_{2}}(\cdot)$ of the channel envelopes, as follows:

$$
M_{\gamma}(s)=\int_{0}^{+\infty} \int_{0}^{+\infty} M_{\gamma}\left(s ; \xi_{1}, \xi_{2}\right) f_{\beta_{1}, \beta_{2}}\left(\xi_{1}, \xi_{2}\right) d \xi_{1} d \xi_{2}
$$

with $M_{\gamma}\left(\cdot ; \beta_{1}, \beta_{2}\right)$ being defined as:

$$
\begin{aligned}
M_{\gamma}\left(s ; \beta_{1}, \beta_{2}\right) & =\left(\frac{1}{2 \pi}\right)^{2} \exp \left(-s \beta_{1}^{2}\right) \exp \left(-s \beta_{2}^{2}\right) \\
& \times \int_{0}^{2 \pi} \int_{0}^{2 \pi} \exp \left[2 s \beta_{1} \beta_{2} \cos \left(\phi_{2}-\phi_{1}\right)\right] d \phi_{1} d \phi_{2}
\end{aligned}
$$

where we have taken into account that $\left\{\varphi_{i}\right\}_{i=1}^{2}$ are independent and uniformly distributed RVs in $[0,2 \pi)$, as described in Section II-B.

By using some algebraic manipulations similar to [17, pp. 339, Eq. (366), Eq. (367)], the two-fold integral in (13) can be computed in closed-form as follows:

$\int_{0}^{2 \pi} \int_{0}^{2 \pi} \exp \left[2 s \beta_{1} \beta_{2} \cos \left(\phi_{2}-\phi_{1}\right)\right] d \phi_{1} d \phi_{2}=(2 \pi)^{2} I_{0}\left(2 s \beta_{1} \beta_{2}\right)$

thus yielding the following expression for $M_{\gamma}\left(\cdot ; \beta_{1}, \beta_{2}\right)$ in (12):

$$
M_{\gamma}\left(s ; \beta_{1}, \beta_{2}\right)=\exp \left(-s \beta_{1}^{2}\right) \exp \left(-s \beta_{2}^{2}\right) I_{0}\left(2 s \beta_{1} \beta_{2}\right)
$$

By substituting (15) in (12), we conclude that the computation of the ABEP requires the integral in (12) to be averaged over the joint distribution of the channel envelopes. Closedform results for (12) are given in the next sub-sections for particular and general fading conditions.

\section{B. ABEP: Independent Fading}

Let us analyze the scenario with uncorrelated fading envelopes, i.e., $\rho_{\beta_{1}^{2} \beta_{2}^{2}}=0$. In this case, $f_{\beta_{1}, \beta_{2}}\left(\xi_{1}, \xi_{2}\right)=$ $f_{\beta_{1}}\left(\xi_{1}\right) f_{\beta_{2}}\left(\xi_{2}\right)$, where $\left\{f_{\beta_{i}}(\cdot)\right\}_{i=1}^{2}$ are the PDFs of univariate Nakagami- $m$ RVs [13, Eq. (2.20)]:

$$
\left\{f_{\beta_{i}}\left(\xi_{i}\right)\right\}_{i=1}^{2}=\tilde{A}_{i} \xi_{i}^{\tilde{C}_{i}} \exp \left(-\tilde{B}_{i} \xi_{i}^{2}\right)
$$

where we have defined $\tilde{A}_{i}=\left(2 m_{i}^{m_{i}}\right) /\left(\Omega_{i}^{m_{i}} \Gamma\left(m_{i}\right)\right), \tilde{B}_{i}=$ $m_{i} / \Omega_{i}$, and $\tilde{C}_{i}=2 m_{i}-1$.

By substituting (16) into (12), the MGF, $M_{\gamma}(\cdot)$, of $\gamma$ simplifies as shown in (17) on top of the next page. In particular, the integral $J(\cdot ; \cdot)$ into the square brackets in (17) can be re-written, by using [19, Eq. (8.4.3.1)], [19, Eq. (8.4.22)] and some algebraic manipulations, as shown in (18) on top of the next page. Furthermore, the integral in (18) can be solved in closed-form by using the Mellin-Barnes theorem in [19, Eq. (2.24.1.1)]:

$$
J\left(s ; \xi_{1}\right)=\frac{1}{2}\left(s+\tilde{B}_{2}\right)^{-\left(\frac{1}{2}+\frac{\tilde{C}_{2}}{2}\right)} G_{1,2}^{1,1}\left(-\frac{s^{2} \xi_{1}^{2}}{s+\tilde{B}_{2}} \mid \begin{array}{c}
\frac{1}{2}-\frac{\tilde{C}_{2}}{2} \\
0
\end{array}\right)_{(19)}
$$

The interested reader might find a good summary of the properties of Meijer-G function and Mellin-Barnes theorem in [27], [28].

Finally, by substituting (19) into (17), and using again [19, Eq. (8.4.3.1)] along with the Mellin-Barnes theorem in [19, Eq. (2.24.1.1)], a closed-form expression for the MGF, $M_{\gamma}(\cdot)$, 


$$
M_{\gamma}(s)=\tilde{A}_{1} \tilde{A}_{2} \int_{0}^{+\infty} \xi_{1}^{\tilde{C}_{1}} \exp \left[-\left(s+\tilde{B}_{1}\right) \xi_{1}^{2}\right] \underbrace{\left[\int_{0}^{+\infty} \xi_{2}^{\tilde{C}_{2}} \exp \left[-\left(s+\tilde{B}_{2}\right) \xi_{2}^{2}\right] I_{0}\left(2 s \xi_{1} \xi_{2}\right) d \xi_{2}\right]}_{J\left(s ; \xi_{1}\right)} d \xi_{1}
$$

$$
J\left(s ; \xi_{1}\right)=\frac{1}{2} \int_{0}^{+\infty} \xi_{2}^{\left(\frac{1}{2} \tilde{C}_{2}-\frac{1}{2}\right)} G_{0,1}^{1,0}\left(\left(s+\tilde{B}_{2}\right) \xi_{2} \mid \begin{array}{l}
- \\
0
\end{array}\right) G_{0,2}^{1,0}\left(\begin{array}{ccc}
-s^{2} \xi_{1}^{2} \xi_{2} \mid & - & 0
\end{array}\right) d \xi_{2}
$$

$$
M_{\gamma}(s)=A \int_{0}^{+\infty} \int_{0}^{+\infty} \xi_{1}^{m} \xi_{2}^{m} \exp \left[-\left(s+B_{1}\right) \xi_{1}^{2}\right] \exp \left[-\left(s+B_{2}\right) \xi_{2}^{2}\right] I_{0}\left(2 s \xi_{1} \xi_{2}\right) I_{m-1}\left(C \xi_{1} \xi_{2}\right) d \xi_{1} d \xi_{2}
$$

of $\gamma$, can be obtained as follows:

$$
\begin{aligned}
& M_{\gamma}(s)=\frac{\tilde{A}_{1} \tilde{A}_{2}}{4}\left(s+\tilde{B}_{1}\right)^{-\left(\frac{1}{2}+\frac{\tilde{C}_{1}}{2}\right)}\left(s+\tilde{B}_{2}\right)^{-\left(\frac{1}{2}+\frac{\tilde{C}_{2}}{2}\right)} \\
& \times G_{2,2}^{1,2}\left(-\frac{s^{2}}{\left(s+\tilde{B}_{1}\right)\left(s+\tilde{B}_{2}\right)} \mid \begin{array}{cc}
\frac{1}{2}-\frac{\tilde{C}_{2}}{2} & \frac{1}{2}-\frac{\tilde{C}_{1}}{2} \\
0 & 0
\end{array}\right)
\end{aligned}
$$

\section{ABEP: Correlated Fading}

Let us analyze the scenario with correlated fading envelopes when $m_{1}=m_{2}=m$. In this case, the channel envelopes, $\left\{\beta_{i}\right\}_{i=1}^{2}$, are distributed according to a bivariate Nakagami- $m$ distribution with joint PDF, $f_{\beta_{1}, \beta_{2}}(\cdot, \cdot)$, as follows [13, Eq. (6.1)]:

$$
\begin{aligned}
f_{\beta_{1}, \beta_{2}}\left(\xi_{1}, \xi_{2}\right) & =A \exp \left(-B_{1} \xi_{1}^{2}\right) \exp \left(-B_{2} \xi_{2}^{2}\right) \\
& \times \xi_{1}^{m} \xi_{2}^{m} I_{m-1}\left(C \xi_{1} \xi_{2}\right)
\end{aligned}
$$

where we have defined:

$$
\left\{\begin{array}{l}
A=\frac{4 m^{m+1}}{\Gamma(m) \Omega_{1} \Omega_{2}\left(1-\rho_{\beta_{1}^{2} \beta_{2}^{2}}\right)\left(\sqrt{\Omega_{1} \Omega_{2} \rho_{\beta_{1}^{2} \beta_{2}^{2}}}\right)^{m-1}} \\
\left\{B_{i}\right\}_{i=1}^{2}=\frac{m}{\Omega_{i}\left(1-\rho_{\beta_{1}^{2} \beta_{2}^{2}}\right)} \\
C=\frac{2 m \sqrt{\rho_{\beta_{1}^{2} \beta_{2}^{2}}}}{\sqrt{\Omega_{1} \Omega_{2}}\left(1-\rho_{\beta_{1}^{2} \beta_{2}^{2}}\right)}
\end{array}\right.
$$

By substituting (21) into (12), the MGF, $M_{\gamma}(\cdot)$, of $\gamma$ simplifies as shown in (23) on top of this page. Then, by using the infinite series representation of the $I_{m-1}(\cdot)$ Bessel function [18, Eq. (9.6.10)] in (23), this latter integral can be re-written as shown in what follows:

$$
M_{\gamma}(s)=\sum_{k=0}^{+\infty}\left[\frac{A C^{m+2 k-1}}{2^{m-1} 4^{k}(k !) \Gamma(k+m)} \Psi_{k}(s)\right]
$$

where $\Psi_{k}(\cdot)$ is defined in (25) on top of the next page.

By using the same analytical steps as for computing $J(\cdot ; \cdot)$ in (17), the integral $\Psi_{k}(\cdot ; \cdot)$ into the square brackets in (25) can be computed in closed-form as follows:

$$
\begin{aligned}
\Psi_{k}\left(s ; \xi_{1}\right) & =\frac{1}{2}\left(s+B_{2}\right)^{-(m+k)} \\
& \times G_{1,2}^{1,1}\left(-\frac{s^{2} \xi_{1}^{2}}{s+B_{2}} \mid \begin{array}{cc}
1-m-k \\
0 & 0
\end{array}\right)
\end{aligned}
$$

Finally, by substituting (26) into (25), and using [19, Eq. (8.4.3.1)] along with the Mellin-Barnes theorem in [19, Eq. (2.24.1.1)], the integral $\Psi_{k}(\cdot)$ can be computed in closedform as shown in what follows:

$$
\begin{aligned}
& \Psi_{k}(s)=\frac{A}{4}\left(s+B_{1}\right)^{-(m+k)}\left(s+B_{2}\right)^{-(m+k)} \\
& \times G_{2,2}^{1,2}\left(-\frac{s^{2}}{\left(s+B_{1}\right)\left(s+B_{2}\right)} \mid \begin{array}{cc}
1-m-k & 1-m-k \\
0 & 0
\end{array}\right)
\end{aligned}
$$

which, along with (24), yields the desired closed-form expression for computing the MGF, $M_{\gamma}(\cdot)$, of $\gamma$.

As a final remark, we observe that, although the final result in (24) requires an infinite series to compute the MGF, this series is absolutely convergent, and converges rapidly thanks to the factorial term and the Gamma function in its denominator, i.e., only a few terms are required to obtain a good accuracy.

\section{ABEP: Correlated Fading With Arbitrary Correlation and Fading Parameters}

Let us now analyze the very general setup with arbitrary correlation and fading parameters. A very general PDF for the bivariate Nakagami- $m$ distribution has been recently introduced in [21], where the limitations of previous models have been overcome. In particular, it has been shown that the most general expression for the PDF of a bivariate Nakagami- $m$ distribution is as follows [21, Eq. (12)] (with $m_{2} \geq m_{1}$ ):

$$
\begin{aligned}
& f_{\beta_{1} \beta_{2}}\left(\xi_{1}, \xi_{2}\right)=f_{\beta_{1}}\left(\xi_{1}\right) f_{\beta_{2}}\left(\xi_{2}\right) \\
& \times \sum_{r=0}^{+\infty} \sum_{k=0}^{r} F(r, k) L_{r+k}^{\left(m_{1}-1\right)}\left(\frac{m_{1}}{\Omega_{1}} \xi_{1}^{2}\right) L_{r+k}^{\left(m_{2}-1\right)}\left(\frac{m_{2}}{\Omega_{2}} \xi_{2}^{2}\right)
\end{aligned}
$$




$$
\Psi_{k}(s)=\int_{0}^{+\infty} \xi_{1}^{2 m+2 k-1} \exp \left[-\left(s+B_{1}\right) \xi_{1}^{2}\right] \underbrace{\left[\int_{0}^{+\infty} \xi_{2}^{2 m+2 k-1} \exp \left[-\left(s+B_{2}\right) \xi_{2}^{2}\right] I_{0}\left(2 s \xi_{1} \xi_{2}\right) d \xi_{2}\right]}_{\Psi_{k}\left(s ; \xi_{1}\right)} d \xi_{1}
$$

$$
M_{\gamma}(s)=\sum_{r=0}^{+\infty} \sum_{k=0}^{r} \sum_{t_{1}=0}^{r+k} \sum_{t_{2}=0}^{r+k}\left[\tilde{A}_{1} \tilde{A}_{2} \tilde{B}_{1}^{t_{1}} \tilde{B}_{2}^{t_{2}} F(r, k) \frac{(-1)^{t_{1}} \Gamma\left(m_{1}+r+k\right)}{t_{1} !\left(r+k-t_{1}\right) ! \Gamma\left(m_{1}+t_{1}\right)} \frac{(-1)^{t_{2}} \Gamma\left(m_{2}+r+k\right)}{t_{2} !\left(r+k-t_{2}\right) ! \Gamma\left(m_{2}+t_{2}\right)} \Upsilon_{t_{1}, t_{2}}(s)\right]
$$

$$
\Upsilon_{t_{1}, t_{2}}(s)=\int_{0}^{+\infty} \int_{0}^{+\infty} \xi_{1}^{\tilde{C}_{1}+2 t_{1}} \xi_{2}^{\tilde{C}_{2}+2 t_{2}} \exp \left[-\left(s+\tilde{B}_{1}\right) \xi_{1}^{2}\right] \exp \left[-\left(s+\tilde{B}_{2}\right) \xi_{2}^{2}\right] I_{0}\left(2 s \xi_{1} \xi_{2}\right) d \xi_{1} d \xi_{1}
$$

$$
\Upsilon_{t_{1}, t_{2}}(s)=\frac{1}{4}\left(s+\tilde{B}_{1}\right)^{-\left(\frac{1}{2}+\frac{\tilde{C}_{1}+2 t_{1}}{2}\right)}\left(s+\tilde{B}_{2}\right)^{-\left(\frac{1}{2}+\frac{\tilde{C}_{2}+2 t_{2}}{2}\right)} G_{2,2}^{1,2}\left(-\frac{s^{2}}{\left(s+\tilde{B}_{1}\right)\left(s+\tilde{B}_{2}\right)} \mid \frac{1}{2}-\frac{\tilde{C}_{1}+2 t_{1}}{0^{2}} \frac{1}{2}-\frac{\tilde{C}_{2}+2 t_{2}}{2}\right)
$$

where:

$$
\begin{aligned}
F(r, k) & =\frac{\left(m_{1} / 2\right)_{r}}{r !}\left(\begin{array}{l}
r \\
k
\end{array}\right)(-1)^{k} \bar{\delta}^{2 k} \tilde{\delta}^{r-k} \\
& \times \frac{(r+k) !}{\left(m_{1} / 2\right)_{r+k}} \frac{(r+k) !}{\left(m_{2} / 2\right)_{r+k}}
\end{aligned}
$$

and $\left\{f_{\beta_{i}}(\cdot)\right\}_{i=1}^{2}$ are defined in (16), $\bar{\delta}=\delta_{1} \delta_{2}-\delta_{3} \delta_{4}$, and $\tilde{\delta}=\delta_{1}^{2}+\delta_{2}^{2}+\delta_{3}^{2}+\delta_{4}^{2}$. In particular, $\left\{\delta_{h}\right\}_{h=1}^{4}$ are the correlation coefficients between the pairs of Rayleigh RVs composing the two Nakagami- $m$ RVs with PDF shown in (28) [21, Fig. 1 and Eq. (2)]. Moreover, the power correlation coefficient is given by $\rho_{\beta_{1}^{2} \beta_{2}^{2}}=0.5 \tilde{\delta} \sqrt{m_{1} / m_{2}}$.

By substituting (16) and (28) into (12) and using [21, Eq. (9)], the MGF, $M_{\gamma}(\cdot)$, of $\gamma$, can be written as shown in (30) on top of this page, where $\Upsilon_{t_{1}, t_{2}}(\cdot)$ is defined in (31) on top of this page. Furthermore, the integral in (31) is similar to (25), and can be computed by using similar analytical steps. In particular, the final result is shown in (32) on top of this page.

We observe that, although the PDF in (28) is the most general expression for the bivariate Nakagami- $m$ distribution, it is written in a very convenient form suitable for further analysis, which has led to (30). Let us also emphasize that, even though, as mentioned in [21], (28) includes as special cases both (16) and (21), we have decided to analyze all cases in order to have simpler final expressions for the spacial cases analyzed in Section III-B and Section III-C. Finally, we also observe that all results above are useful for any values of the fading parameters $\left\{m_{i}\right\}_{i=1}^{2}$, which, in particular, are not restricted to be integer or half-integer values [21].

\section{ABEP Over CorRelated NaKagami- $m$ Fading Channels: The $N_{t} \times 1$ Miso Setup}

In Section III, we have provided exact, even though expressed in the form of a single-integral to be computed numerically, expressions for the ABEP when the transmitter is equipped with two transmit-antennas. In this section, the frameworks are generalized to account for an arbitrary number of antennas at the transmitter. In particular, we will propose two tight upper bounds for computing the ABEP in closedform, and will show in Section $V$ the accuracy of them for various system setups and channel conditions. We have decided to resort to bounds for two main reasons. i) To avoid the need to manage the multivariate Nakagami- $m$ distribution, whose PDF, besides having some constraints on the structure of the correlation matrix [22], [23], is much more complicated than the bivariate case. ii) Our main goal is to develop a framework that can be used for a quick analysis and design of MISO systems adopting SSK modulation. The development of a framework involving the multivariate Nakagami$m$ distribution would inevitably lead to final expressions that would be more complicated than those already developed in Section III. Moving from these two considerations, we propose two asymptotically tight upper bounds that only need the error probabilities between pairs of generically correlated links to be computed. This way, two benefits will be achieved: i) the final framework will have a complexity comparable to the system setup with $N_{t}=2$, and ii) the final framework will be useful for arbitrary correlation functions and fading parameters in all wireless links.

1) Symbol-based Union Bound: The first bound, which is called Symbol-based Union Bound (SUB), can be obtained by using typical methods for performance analysis of multi-level modulation schemes with optimum detection [29, Sec. 5.7]. In particular, for an arbitrary number of transmit-antennas $N_{t}$, each antenna index at the transmitter is unambiguously identified by a sequence of $\log _{2}\left(N_{t}\right)$ bits. Accordingly, the SUB can be obtained in two steps: i) first, the average error probability of incorrect detection of the active antenna at the transmitter, i.e., the so-called Average Constellation Error Probability (ACEP), is upper bounded, and ii) second, the ABEP is obtained from the ACEP by taking into account that 


$$
\mathrm{ABEP} \leq \mathrm{ABEP}{ }^{\mathrm{CUB}}=\frac{1}{\log _{2}\left(N_{t}\right)} \frac{1}{N_{t}} \sum_{i_{1}=1}^{N_{t}} \sum_{i_{2} \neq i_{1}=1}^{N_{t}} N_{b}\left(i_{1}, i_{2}\right) \operatorname{PEP}\left(\mathrm{TX}_{i_{1}} \rightarrow \mathrm{TX}_{i_{2}}\right)
$$

the errors for all antenna indexes are equally likely.

In formulas, the ACEP can be upper bounded via union bound techniques [29, Eq. (5.86)], as follows:

$$
\mathrm{ACEP} \leq \frac{1}{N_{t}} \sum_{i_{1}=1}^{N_{t}} \sum_{i_{1} \neq i_{2}=1}^{N_{t}} \operatorname{PEP}\left(\mathrm{TX}_{i_{1}} \rightarrow \mathrm{TX}_{i_{2}}\right)
$$

where PEP $\left(\mathrm{TX}_{i_{1}} \rightarrow \mathrm{TX}_{i_{2}}\right)$ denotes the Pairwise Error Probability (PEP) of the transmit-antennas $\mathrm{TX}_{i_{1}}$ and $\mathrm{TX}_{i_{2}}$ with $i_{1}, i_{2}=1,2, \ldots, N_{t}$, i.e., the probability of detecting $\mathrm{TX}_{i_{2}}$ when, instead, $\mathrm{TX}_{i_{1}}$ is actually transmitting. In particular, $\operatorname{PEP}\left(\mathrm{TX}_{i_{1}} \rightarrow \mathrm{TX}_{i_{2}}\right)$ is the ABEP of an equivalent $2 \times 1$ MISO system where only the transmit-antennas $\mathrm{TX}_{i_{1}}$ and $\mathrm{TX}_{i_{2}}$ can be activated for transmission. In other words, $\mathrm{PEP}\left(\mathrm{TX}_{i_{1}} \rightarrow \mathrm{TX}_{i_{2}}\right)$ in (33) is the ABEP computed in Section III when $N_{t}=2$, i.e., (11).

From the ACEP in (33), the ABEP for an arbitrary number of transmit-antennas can be upper bounded by using the method described in [29, Eq. (5.101)]. In particular, let us assume that all transmit-antenna index errors are equally likely. In such a case, each transmit-antenna index error will occur with the same probability equal to $\operatorname{ACEP} /\left(N_{t}-1\right)$. Since there are $N_{t} / 2$ cases of transmit-antenna index errors for any particular bit in each sequence of $\log _{2}\left(N_{t}\right)$ bits, then the ABEP can be upper bounded as follows [29, Eq. (5.101)]:

$$
\mathrm{ABEP} \leq \mathrm{ABEP}^{\mathrm{SUB}}=\frac{N_{t} / 2}{N_{t}-1} \mathrm{ACEP}
$$

2) Codeword-based Union Bound: The second bound, which is called Codeword-based Union Bound (CUB), can be obtained by using typical methods for performance analysis of Maximum Likelihood Sequence Estimation (MLSE) detectors [13, Sec. 13.1.3]. In particular, an upper bound for the ABEP can be obtained from [13, Sec. 13.44], as shown in (35) on top of this page, where $N_{b}\left(i_{1}, i_{2}\right)$ is the number of information bit errors committed by choosing $\mathrm{TX}_{i_{2}}$ instead of $\mathrm{TX}_{i_{1}}$ as transmit-antenna. Moreover, we have taken into account the following facts: i) in an uncoded system, like the one we are considering in this paper, the number of information bits per transmission is $\log _{2}\left(N_{t}\right)$, and ii) the random properties of the binary source at the transmitter results in activating the transmit-antennas with equal probability, i.e., $1 / N_{t}$.

\section{3) Comparison With other Bounds for SSK Modulation:}

Let us compare the bounds proposed in (34) and (35) with other bounds already available in the literature for analyzing the performance of SSK modulation. The bound in (35) seems to be similar, even though different, to [6, Eq. (4)]. They differ by a constant factor $1 / \log _{2}\left(N_{t}\right)$, which is not present in $[6$, Eq. (4)]. In [6], it is shown that [6, Eq. (4)] can be very weak and is not, in general, asymptotically tight. To overcome this problem, an $a d-h o c$ bound is proposed for Rayleigh fading channels [6, Eq. (9)], where a correction term needs to be introduced in order to obtain the desired accuracy for large Signal-to-Noise Ratios (SNRs). A closed-form expression for this correction term is not given in [6]: it has to be computed for each $N_{t}$ and fading distribution. The bound in (35) offers a simple way to overcome the problems in [6], by avoiding the $a d-h o c$ adaptation of [6, Eq. (4)] and yielding, at the same time, tight estimates in the high SNR regime.

On the other hand, to the best of the authors knowledge, bounds similar to (34) are not available in the literature for computing the performance of SSK modulation for an arbitrary number of transmit-antennas. In Section V, we will show that both bounds offer almost the same accuracy, with (34) being simpler than (35) to be computed, given that it does not require the knowledge of $N_{b}(\cdot, \cdot)$. Moreover, since these bounds are all based on the frameworks developed in Section III, there are not limitations to apply them for any fading scenario with arbitrary correlation, fading parameters, and power imbalance among all wireless links of the MISO system.

\section{Numerical and Simulation Results}

In this section, we provide some numerical results with a twofold objective: i) to validate the accuracy of the analytical frameworks developed in Section III and Section IV, and ii) to analyze the performance of SSK modulation for different fading scenarios, i.e., fading correlation, fading parameters, and power imbalance among the wireless links (i.e., $\left\{\Omega_{i}\right\}_{i=1}^{N_{t}}$ are different among the wireless links). Since various fading conditions are analyzed, the system setup used to obtain the numerical examples is shown for each figure in its caption. Monte Carlo simulations are obtained by using the simulation framework proposed in [30] ${ }^{4}$ and [21] to generate bivariate Nakagami- $m$ fading envelopes with the PDF shown in (21) and (28), respectively. On the other hand, when $N_{t}>2$ Monte Carlo simulations are obtained by using the simulation framework introduced in [31] to generate multivariate Nakagami- $m$ fading envelopes. Moreover, the series in (24) is truncated to the first 20 terms to get very accurate numerical estimates, while the series in (30) is truncated to the first 5 terms. Finally, from the MGFs in (20), (24), and (30), the ABEP is obtained from (11) by using straightforward numerical integration techniques.

a) $2 \times 1$ MISO System with Uncorrelated Fading: In Fig. 1 and Fig. 2, the scenario with uncorrelated fading for a balanced and an unbalanced setup is shown, respectively. By comparing the two figures, the following observations can be made: i) the proposed analytical model is very accurate and well overlaps with Monte Carlo simulations for various system settings, ii) the system performance improves for unbalanced fading as a result of (10), which shows that the system performance depends on the difference between the complex fading gains of the two wireless links, iii) when a balanced

\footnotetext{
${ }^{4}$ Note that some typos in [30] have been adequately fixed and carefully taken into account to produce Monte Carlo simulation results. These typos and corrections are listed in Appendix A.
} 


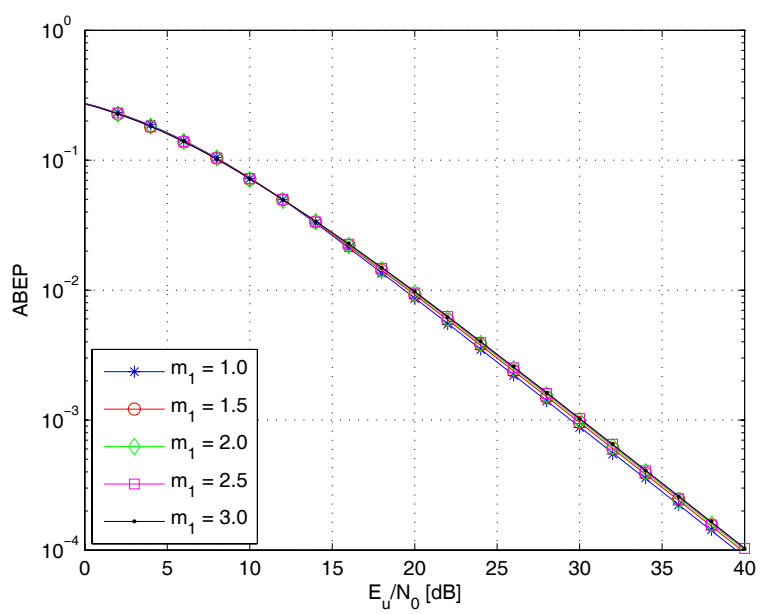

Fig. 1. Comparison between Monte Carlo simulation (markers) and analytical model (solid lines). $2 \times 1$ MISO system. Uncorrelated fading model in (16) (i.e., $\rho_{\beta_{1}^{2} \beta_{2}^{2}}=0$ ) with balanced power (i.e., $\Omega_{1}=\Omega_{2}=1$ ), and $m_{2}=2$.

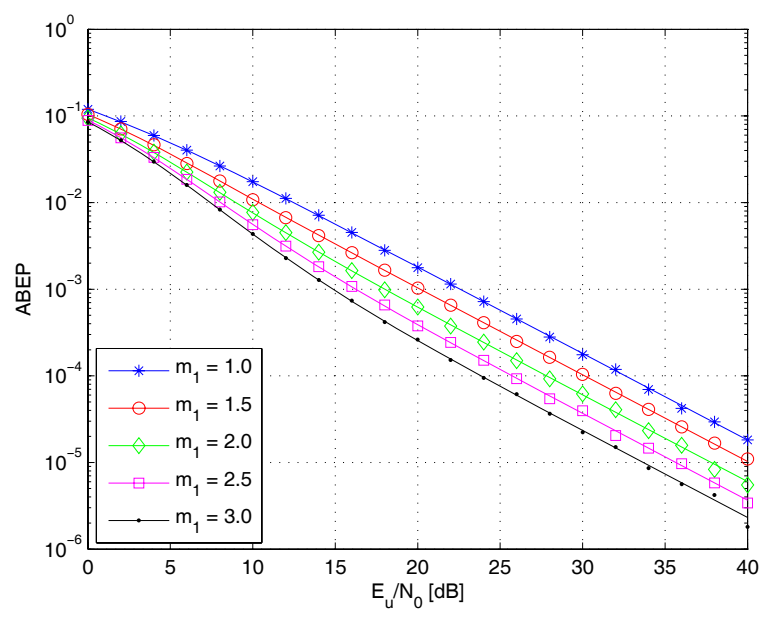

Fig. 2. Comparison between Monte Carlo simulation (markers) and analytical model (solid lines). $2 \times 1 \mathrm{MISO}$ system. Uncorrelated fading model in (16) (i.e., $\rho_{\beta_{1}^{2} \beta_{2}^{2}}=0$ ) with unbalanced power (i.e., $\Omega_{1}=10 \Omega_{2}$ ), and $m_{2}=2$, $\Omega_{2}=1$.

fading scenario is considered, the system performance is almost the same for various Nakagami- $m$ fading parameters, i.e., the ABEP changes very little with the fading severity, and iv) on the other hand, for unbalanced fading the system performance improves significantly for less severe fading conditions (i.e., $m_{1}$ increases). We also remark that similar conclusions, both qualitative and quantitative, can be drawn for different values of $m_{2}$, even though the results are not shown in this manuscript due to space constraints.

With regard to the performance comparison between balanced and unbalanced setups, we emphasize here that the setups in Fig. 1 and Fig. 2 have a different average SNR per branch. As a consequence, the better performance achieved by the unbalanced setup with respect to the balanced setup is due, in part, to this latter aspect. To analyze the performance of two similar systems but with the same average SNR per branch in the balanced and unbalanced configurations, the curves in Fig. 1 should be shifted on the left by $10 \log _{10}(5.5) \cong 7.4 \mathrm{~dB}$.
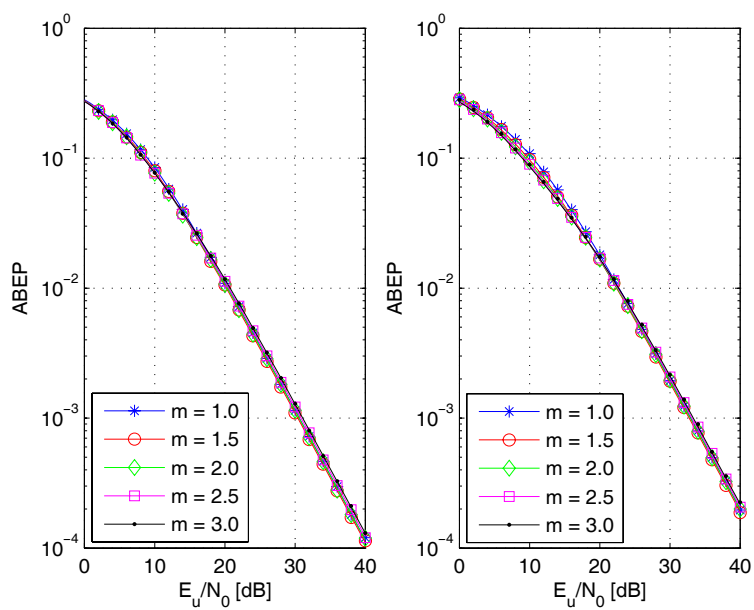

Fig. 3. Comparison between Monte Carlo simulation (markers) and analytical model (solid lines). $2 \times 1$ MISO system. Correlated fading model in (21) (i.e., $\rho_{\beta_{1}^{2} \beta_{2}^{2}}=0.25$ on the left and $\rho_{\beta_{1}^{2} \beta_{2}^{2}}=0.75$ on the right) with balanced power (i.e., $\Omega_{1}=\Omega_{2}=1$ ).

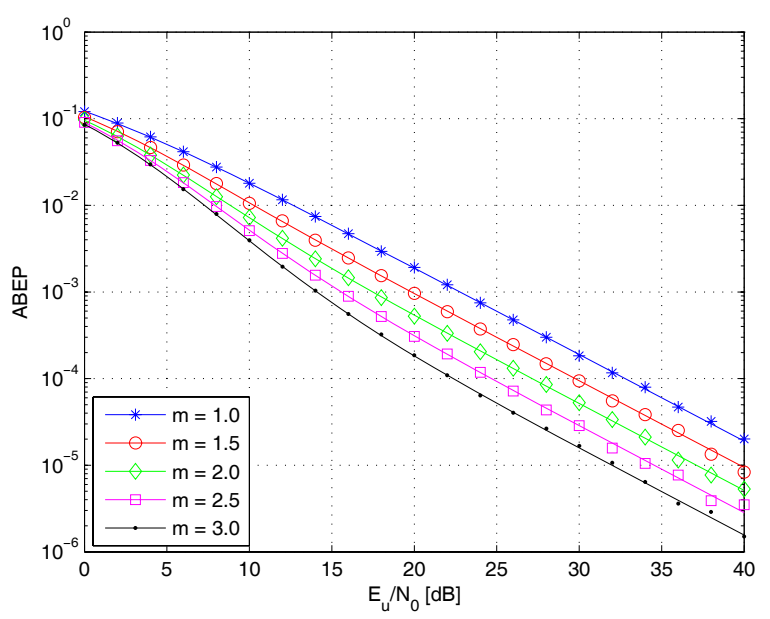

Fig. 4. Comparison between Monte Carlo simulation (markers) and analytical model (solid lines). $2 \times 1$ MISO system. Correlated fading model in (21) (i.e., $\rho_{\beta_{1}^{2} \beta_{2}^{2}}=0.25$ ) with unbalanced power (i.e., $\Omega_{1}=10 \Omega_{2}$ ), and $\Omega_{2}=1$.

By doing so, we realize that if $m_{1}=1$ there is almost no difference between balanced and unbalanced setups, but we can notice a significant performance improvement in the unbalanced setting if $m_{1}=3$. As mentioned above, this is confirmed by the analytical framework in (10). A similar comment and left-shifting operation should be applied to several other curves shown in this paper if the reader is interested in comparing system setups with the same average SNR per branch. Finally, we emphasize here that power imbalance might arise in several cases and for many different reasons (see, e.g., [32], [33]).

b) $2 \times 1$ MISO System with Correlated Fading in (21):

In Figs. 3-5, the scenario with correlated fading for a low and high correlation coefficient, and balanced and unbalanced fading is shown. By carefully analyzing the figures, the following observations can be made: i) also in this case, the proposed analytical model is very accurate and well overlaps with Monte Carlo simulations for various system settings, ii) 


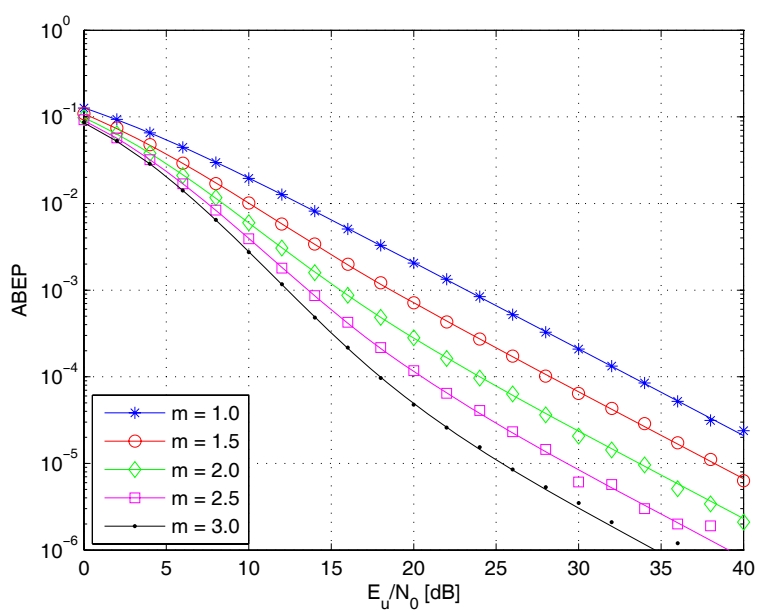

Fig. 5. Comparison between Monte Carlo simulation (markers) and analytical model (solid lines). $2 \times 1$ MISO system. Correlated fading model in (21) (i.e., $\rho_{\beta_{1}^{2} \beta_{2}^{2}}=0.75$ ) with unbalanced power (i.e., $\Omega_{1}=10 \Omega_{2}$ ), and $\Omega_{2}=1$.

similar to the uncorrelated scenario, SSK modulation offers better performance for unbalanced fading conditions, since, in this case, the multipath channels are more distinguishable from each other, iii) for a balanced fading setup, i.e., $\Omega_{1}=\Omega_{2}$, we note that the performance gets worse as the correlation coefficient increases, as expected; moreover, we see that the performance is almost the same for different values of the fading severity $(m)$, and iv) on the contrary, for an unbalanced fading setup, the system behavior is very different: the performance improves when the fading is less severe (a result similar to the uncorrelated system setup), but, very surprisingly, the system performance gets better as the correlation coefficient between the links increases. This latter and apparently unexpected result can be explained as follows. When the wireless links are unbalanced and strongly correlated two favorable conditions for SSK modulation are verified simultaneously: i) the links are more distinguishable from each other due to the unbalanced powers, and ii) the fading fluctuations of each of them change jointly. As a consequence, the fading fluctuations, especially for less severe fading, cannot alter significantly the average power gap (i.e., $\Omega_{1} \neq \Omega_{2}$ ) between them, thus yielding good system performance. On the other hand, when the links are uncorrelated, but still unbalanced, the links are subject to independent fading fluctuations, which can induce more pronounced variations with respect to the average value, thus yielding worse performance. In other words, deep fades are more likely to offset the average power gap in this case.

c) $2 \times 1$ MISO System with Correlated Fading in (28): In Fig. 6, we analyze the system performance when the correlated fading model in (28) is considered. Besides the good agreement between analytical model and Monte Carlo simulations, as well as the improved performance for unbalanced settings, we observe that the ABEP changes significantly when, still having the same power imbalance ratio (i.e., $\Omega_{2} / \Omega_{1}$ ), the correlation coefficient and the fading severity are different. This latter phenomenon can be observed by comparing Scenario $b$ and Scenario $c$ in Fig. 6, where a non-negligible performance gap is observable. This result

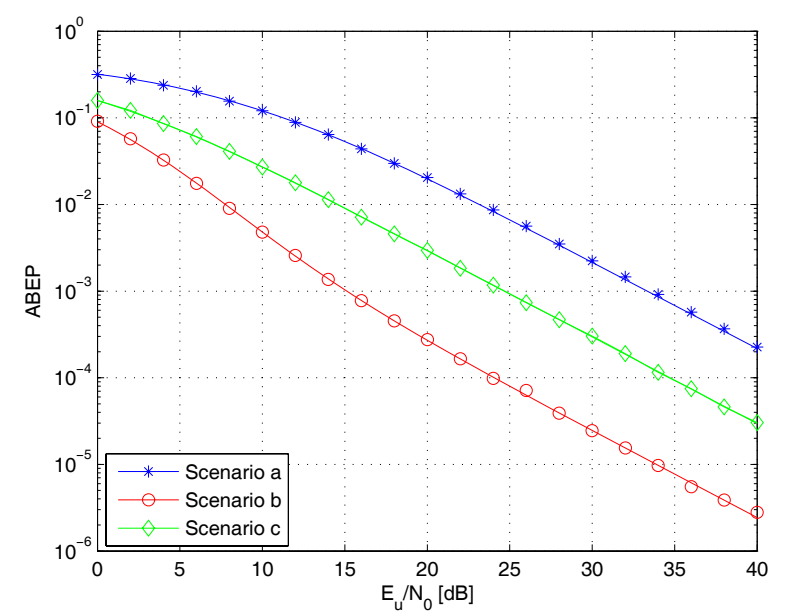

Fig. 6. Comparison between Monte Carlo simulation (markers) and analytical model (solid lines). $2 \times 1$ MISO system. Correlated fading model in (28). Scenario a: $m_{1}=m_{2}=1, \Omega_{1}=\Omega_{2}=2 / 3, \delta_{1}=0.50, \delta_{2}=\delta_{3}=\delta_{4}=$ 0.45 . Scenario b: $m_{1}=1, m_{2}=2.5, \Omega_{1}=1, \Omega_{2}=10, \delta_{1}=\delta_{2}=\delta_{3}=$ $0.45, \delta_{4}=-0.45$. Scenario c: $m_{1}=m_{2}=1, \Omega_{1}=2 / 3, \Omega_{2}=20 / 3$, $\delta_{1}=0.50, \delta_{2}=\delta_{3}=\delta_{4}=0.45$.

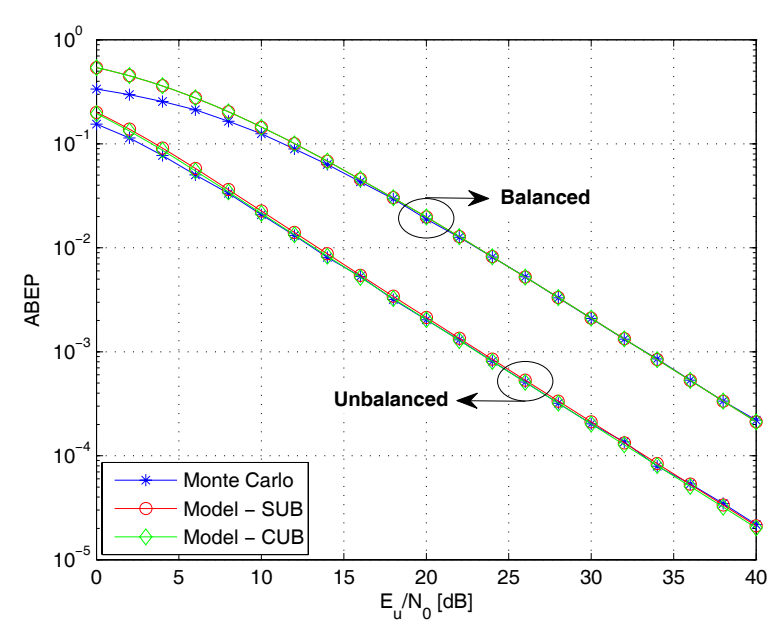

Fig. 7. Comparison between Monte Carlo simulation and analytical model. $4 \times 1$ MISO system. Uncorrelated fading model in (16) (i.e., model. $\left.4 \rho_{\beta_{i}^{2} \beta_{j}^{2}}\right\}_{i \neq j=1}^{4}=0$ ) with balanced (i.e., $\left\{\Omega_{i}\right\}_{i=1}^{4}=1$ ) and unbalanced (i.e., $\Omega_{1}=1,\left\{\Omega_{i}\right\}_{i=2}^{4}=4(i-1)$ ) power, and $m=2.5$.

confirms that the performance of SSK modulation is strongly affected by the characteristics of the wireless channel and the fading distribution as well, since Scenario $c$ is representative of a Rayleigh fading channel. Note that this non-negligible performance gap is kept when the curve showing Scenario $c$ is left-shifted by $10 \log _{10}(3 / 2)=1.76 \mathrm{~dB}$ for analyzing two system setups with the same average SNR per branch. Finally, from the numerical results described so far, we can readily observe that the worst-case scenario for the ABEP is given by the balanced case with high-correlated wireless links. This is also confirmed by (10).

d) $4 \times 1$ and $8 \times 1$ MISO Systems with Uncorrelated Fading: In Figs. 7-9, the ABEP for a MISO system with $N_{t}>2$ is shown and both bounds proposed in Section IV are compared with Monte Carlo simulations. We note that 


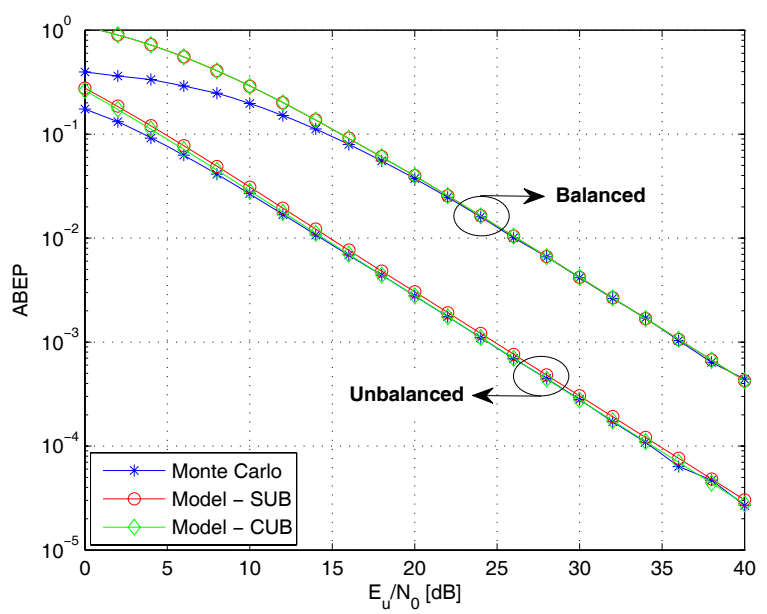

Fig. 8. Comparison between Monte Carlo simulation and analytical model. $8 \times 1$ MISO system. Uncorrelated fading model in (16) (i.e., $\left\{\rho_{\beta_{i}^{2} \beta_{j}^{2}}\right\}_{i \neq j=1}^{8}=0$ ) with balanced (i.e., $\left\{\Omega_{i}\right\}_{i=1}^{8}=1$ ) and unbalanced (i.e., $\Omega_{1}=1,\left\{\Omega_{i}\right\}_{i=2}^{8}=3(i-1)$ ) power, and $m=2.5$.

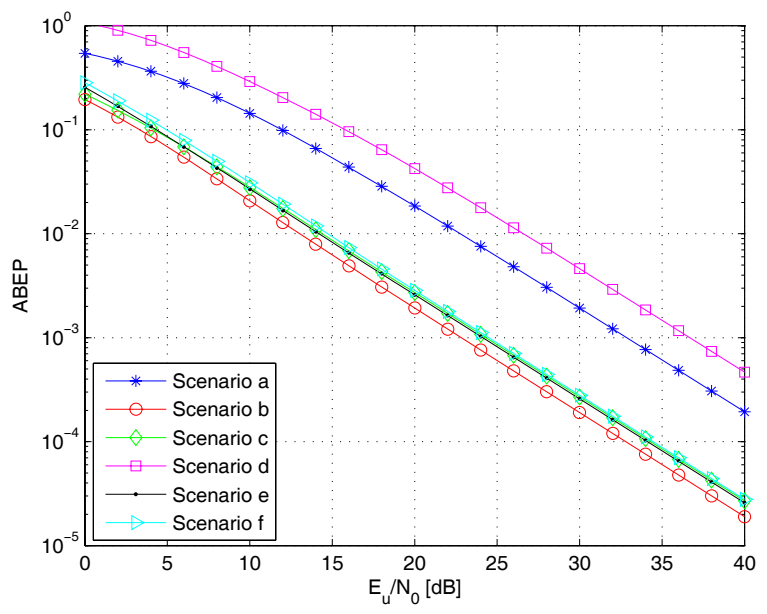

Fig. 9. Performance comparison (analytical model only) between $4 \times 1$ and $8 \times 1$ MISO systems for various fading scenarios. Uncorrelated fading model in (16) (i.e., $\left\{\rho_{\beta_{i}^{2} \beta_{j}^{2}}\right\}_{i \neq j=1}^{8}=0$ ). Scenario a: $N_{t}=4,\left\{m_{i}\right\}_{i=1}^{N_{t}}=i$, $\left\{\Omega_{i}\right\}_{i=1}^{N_{t}}=1$. Scenario b: $N_{t}=4,\left\{m_{i}\right\}_{i=1}^{N_{t}}=i, \Omega_{1}=1,\left\{\Omega_{i}\right\}_{i=2}^{N_{t}}=$ $4(i-1)$. Scenario c: $N_{t}=4,\left\{m_{i}\right\}_{i=1}^{N_{t}}=5-i, \Omega_{1}=1,\left\{\Omega_{i}\right\}_{i=2}^{N_{t}}=$ $4(i-1)$. Scenario d: $N_{t}=8,\left\{m_{i}\right\}_{i=1}^{N_{t}}=i,\left\{\Omega_{i}\right\}_{i=1}^{N_{t}}=1$. Scenario e: $N_{t}=8,\left\{m_{i}\right\}_{i=1}^{N_{t}}=i, \Omega_{1}=1,\left\{\Omega_{i}\right\}_{i=2}^{N_{t}}=3(i-1)$. Scenario f: $N_{t}=8$, $\left\{m_{i}\right\}_{i=1}^{N_{t}}=9-i, \Omega_{1}=1,\left\{\Omega_{i}\right\}_{i=2}^{N_{t}}=3(i-1)$.

both bounds are very accurate, asymptotically tight, and offer almost the same accuracy for various system settings and fading conditions. Moreover, by carefully analyzing Fig. 9, we note that: i) by comparing Scenario a with Scenario $d$, we note that the ABEP degrades when $N_{t}$ increases, which agrees with [6], and ii) by comparing Scenario $b$ with Scenario $c$ and Scenario e with Scenario $f$, we observe that, for this particular setup, different values of the fading severity, i.e., $\left\{m_{i}\right\}_{i=1}^{N_{t}}$, do not modify significantly the system performance. Similar to previous figures, the comparison for system setups with the same average SNR per branch can be obtained by left-shifting operations.

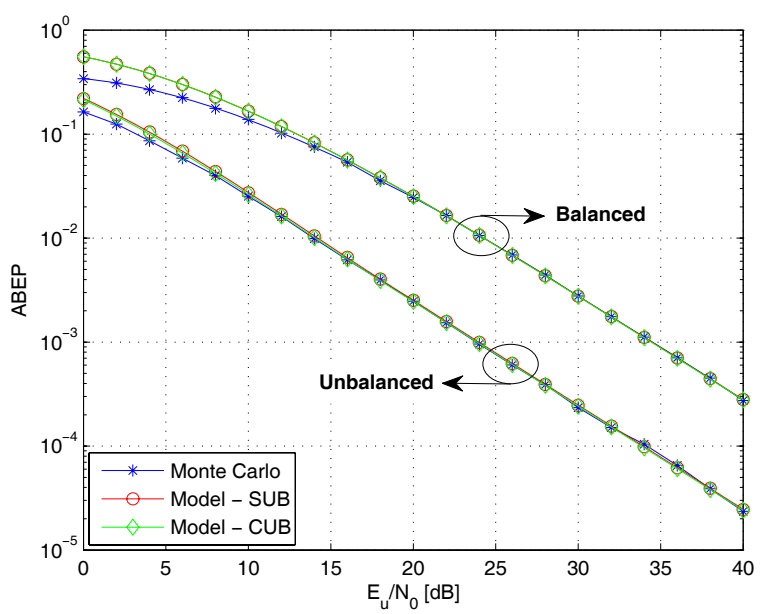

Fig. 10. Comparison between Monte Carlo simulation and analytical model. $4 \times 1$ MISO system. Correlated fading model in (21) (i.e., $\left\{\rho_{\beta_{i}^{2} \beta_{j}^{2}}\right\}_{i, j=1}^{4} \cong$ $\left.\left\{\rho_{\beta_{i} \beta_{j}}\right\}_{i, j=1}^{4}=\exp (-0.5|i-j|)\right)$ with balanced (i.e., $\left\{\Omega_{i}\right\}_{i=1}^{4}=1$ ) and unbalanced (i.e., $\Omega_{1}=1,\left\{\Omega_{i}\right\}_{i=2}^{4}=4(i-1)$ ) power, and $\left\{m_{i}\right\}_{i=1}^{4}=$ 2 .

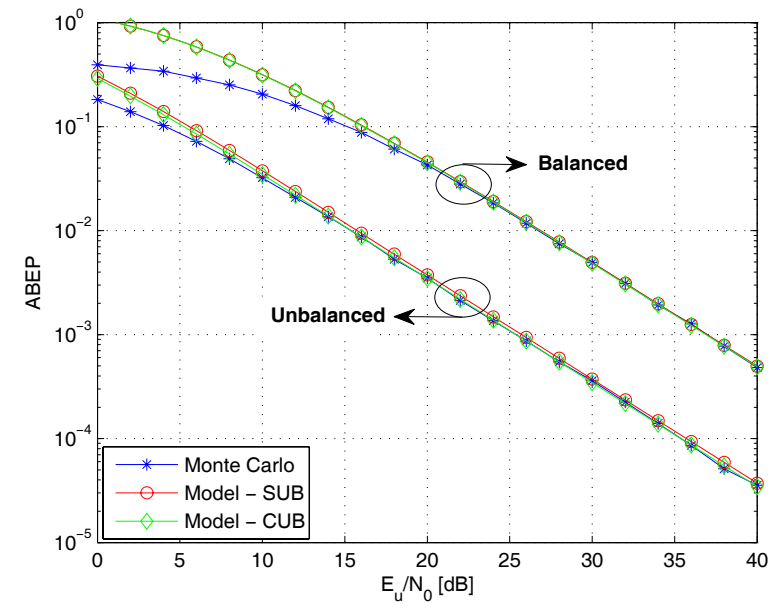

Fig. 11. Comparison between Monte Carlo simulation and analytical model. $8 \times 1$ MISO system. Correlated fading model in (21) (i.e., $\left\{\rho_{\beta_{i}^{2} \beta_{j}^{2}}\right\}_{i, j=1}^{8} \cong$ $\left.\left\{\rho_{\beta_{i} \beta_{j}}\right\}_{i, j=1}^{8}=\exp (-0.5|i-j|)\right)$ with balanced (i.e., $\left.\left\{\Omega_{i}\right\}_{i=1}^{8}=1\right)$ and unbalanced (i.e., $\Omega_{1}=1,\left\{\Omega_{i}\right\}_{i=2}^{8}=3(i-1)$ ) power, and $\left\{m_{i}\right\}_{i=1}^{8}=$ 2 .

e) $4 \times 1$ and $8 \times 1$ MISO Systems with Correlated Fading in (21): Finally, in Figs. 10-12 we analyze the scenario with correlated fading. In particular, as shown in the captions of the figures, an exponential correlation profile has been considered in order to take into account that closely-spaced transmitantennas have a higher spatial correlation. In particular, also in this case, Fig. 10 and Fig. 11 confirm that the analytical framework is very accurate and asymptotically exact for large SNRs. Moreover, a negligible difference is observable between the two bounds. Interesting results can be obtained from Fig. 12. In particular, when $N_{t}=8$, we have considered two different correlation patterns: i) in Scenario c and Scenario $d$, the largest value of the correlation coefficient is the same 


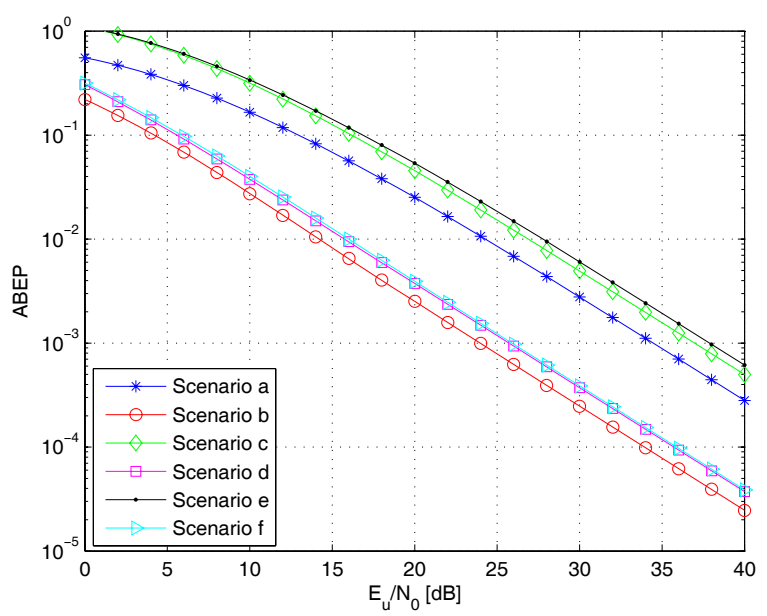

Fig. 12. Performance comparison (analytical model only) between $4 \times 1$ and $8 \times 1$ MISO systems for various fading scenarios. Correlated fading model in (21). Common setting: $\left\{m_{i}\right\}_{i=1}^{N_{t}}=2$ and $\left\{\rho_{\beta_{i}^{2} \beta_{j}^{2}}\right\}_{i, j=1}^{N_{t}} \cong$ $\left\{\rho_{\beta_{i} \beta_{j}}\right\}_{i, j=1}^{N_{t}}=\exp \left(-d_{0}|i-j|\right)$. Scenario a: $N_{t}=4, d_{0}=0.5$, $\left\{\Omega_{i}\right\}_{i=1}^{N_{t}}=1$. Scenario b: $N_{t}=4, d_{0}=0.5, \Omega_{1}=1,\left\{\Omega_{i}\right\}_{i=2}^{N_{t}}=$ $4(i-1)$. Scenario c: $N_{t}=8, d_{0}=0.5,\left\{\Omega_{i}\right\}_{i=1}^{N_{t}}=1$. Scenario d: $N_{t}=8, d_{0}=0.5, \Omega_{1}=1,\left\{\Omega_{i}\right\}_{i=2}^{N_{t}}=3(i-1)$. Scenario e: $N_{t}=8$, $d_{0}=0.25,\left\{\Omega_{i}\right\}_{i=1}^{N_{t}}=1$. Scenario f: $N_{t}=8, d_{0}=0.25, \Omega_{1}=1$, $\left\{\Omega_{i}\right\}_{i=2}^{N_{t}}=3(i-1)$.

as that of the system setup with $N_{t}=4$ (i.e., $d_{0}=0.5$ ), and ii) in Scenario $e$ and Scenario $f$, the largest value of the correlation coefficient is greater than that of the system setup with $N_{t}=4$ (i.e., $d_{0}=0.25$ ). Case study i) corresponds to a physical scenario in which we have moved from four to eight antennas by increasing the geometric size of the array (e.g., let us consider a linear array for illustrative purposes). On the other hand, case study ii) corresponds to a physical scenario in which we have moved from four to eight antennas by approximately maintaining the geometric size of the array, thus resulting in more closely-spaced, and so correlated, transmitantennas. In Fig. 12, we notice that the ABEP of both case studies is almost the same.

f) Closing Comments: In conclusions, the results shown in Figs. 1-12 clearly point out that the performance of SSK modulation is significantly affected by the fading scenario. In particular, we have noticed that the performance of SSK modulation might improve, for some fading scenarios, in the presence of power imbalance. In this paper, power imbalance is only due to the wireless channel, since all antennas are assumed to transmit the same average power when they are activated for data transmission. However, unbalanced system setups can be artificially created by allowing each antenna to transmit a different power, according to the fading conditions in each wireless link. This remark follows directly by considering, e.g., the MISO system with $N_{t}=2$ and by taking into account that when the average energies transmitted by the antennas are different, i.e., $E_{u_{1}} \neq E_{u_{2}}$, the error probability in (10) can be generalized as follows:

$$
\mathrm{P}_{\mathrm{E}}\left(h_{1}, h_{2}\right)=Q\left(\sqrt{\frac{1}{4 N_{0}}\left|\sqrt{E_{u_{2}}} \alpha_{2}-\sqrt{E_{u_{1}}} \alpha_{1}\right|^{2}}\right)
$$

which clearly highlights that the condition, e.g., $E_{u_{2}}>E_{u_{1}}$ can lead to unbalanced wireless links even though $\mathrm{E}\left\{\beta_{1}^{2}\right\} \cong$ $\mathrm{E}\left\{\beta_{2}^{2}\right\}$.

In other words, the results shown in this paper suggest that the system performance might be improved by adopting some opportunistic power allocation mechanisms to allow an easier differentiation among the wireless links. In this regard, the analytical frameworks developed in this paper could be the enabling analytical tool for a systematic system optimization of SSK modulation over realistic fading conditions. Some preliminary results showing the potential advantages of opportunistic power allocation methods for SSK modulation can be found in [34] for a simple $2 \times 1$ MIMO scheme over correlated Rayleigh fading channels.

\section{CONClusion}

In this paper, we have proposed an accurate framework for analyzing the performance of SSK modulation over correlated Nakagami- $m$ fading channels with arbitrary correlation and fading parameters. Numerical results have validated the accuracy of the proposed analytical derivation, and shown that the system performance can change remarkably for different fading conditions. In particular, for some fading scenarios, we have noticed that power imbalance among the wireless links might offer better performance. Overall, the results shown in this paper suggest that SSK modulation can be a suitable transmission technology for MIMO systems, and, in particular, for distributed MIMO settings where the transmitantennas could be geographically far apart from each other: a scenario where the transmit-receive wireless links are likely to be subject to unbalanced fading, besides being subject to uncorrelated fading fluctuations.

Our current research activity is concerned with the extension of the framework proposed in this paper for SSK modulation to the more general SM scheme. Some results for uncorrelated fading and real signal constellations are already available in the literature (see, e.g., [2], [5]). However, the analysis of arbitrary modulation schemes appear to be a more complicated analytical problem, even for uncorrelated fading channels [5], which deserves further attention.

\section{ACKNOWLEDGMENT}

We gratefully acknowledge support from the EPSRC (EP/G011788/1) for this work. Harald Haas acknowledges the Scottish Funding Council support of his position within the Edinburgh Research Partnership in Engineering and Mathematics between the University of Edinburgh and Heriot Watt University.

\section{APPENDIX A TYPOS AND CORRECTIONS IN [30]}

The following typos can be found in [30, Sec. 2.1]. The notation is introduced in the original paper [30].

- [30, Eq. 10] should be replaced by:

$$
\sigma=\sqrt{\frac{1-\rho}{2 m} \Omega_{2}}
$$


This result can be obtained by comparing [30, Eq. 5] and [30, Eq. 6].

- [30, Eq. 17] and, so [30, Eq. 14], needs to be replaced by:

$$
x_{i} \sim N_{m}\left(r_{0}, \sigma\right) \approx M\left(m, 2 m \sigma^{2}\right)
$$

The result in (38) can be obtained by using the infinite series representation of the modified Bessel function of first kind, $I_{v}(\cdot)$, in $[18,9.6 .10]$. In detail, by truncating $[18,9.6 .10]$ to the first term and substituting it into [30, Eq. (6)], (38) can be derived via direct comparison with [30, Eq. (4)].

Let us also note that [35, Eq. (70)] mentioned in [30, Appendix] is correct owing to the different parametrization in [35, Eq. (66)] and [30, Eq. (6)].

- In [30, Fig. 1], when generating $r_{22}, \sigma$ needs to be replaced by:

$$
\sigma=\sqrt{\frac{1-\rho}{2 m} \Omega_{2}}
$$

Let us emphasize that, even though $r_{22}$ takes into account only the integer part of the fading parameter $m, \sigma$ actually depends on $m$ and not on $\lfloor m\rfloor$ only. This follows directly from the application of Property 1 and Property 3 in [30, Appendix].

- In [30, Fig. 1], $r_{22}$ needs to be replaced by:

$$
r_{22}=\sqrt{\sum_{i=1}^{\lfloor m\rfloor} x_{i}^{2}}
$$

This follows from Property 1 in [30, Appendix].

- In [30, Fig. 1], $r_{21}$ needs to be replaced by:

$$
r_{21}=\sqrt{2 \sigma^{2} s_{21}}
$$

which follows from (38). We also emphasize that $\sigma$ in (41) can be obtained from (39).

\section{REFERENCES}

[1] J. Mietzner, R. Schober, L. Lampe, W. H. Gerstacker, and P. A. Hoeher, "Multiple-antenna techniques for wireless communications-a comprehensive literature survey," IEEE Commun. Surveys \& Tutorials, vol. 11, no. 2, pp. 87-105, 2nd quarter 2009.

[2] Y. Chau and S.-H. Yu, "Space modulation on wireless fading channels," in Proc. IEEE Veh. Technol. Conf.-Fall, Oct. 2001, vol. 3, pp. 1668 1671.

[3] H. Haas, E. Costa, and E. Schultz, "Increasing spectral efficiency by data multiplexing using antennas arrays," in Proc. IEEE Int. Symp. Personal, Indoor, Mobile Radio Commun., Sep. 2002, vol. 2, pp. 610-613.

[4] R. Y. Mesleh, H. Haas, S. Sinanovic, C. W. Ahn, and S. Yun, "Spatial modulation," IEEE Trans. Veh. Technol., vol. 57, no. 4, pp. 2228-2241, July 2008.

[5] J. Jeganathan, A. Ghrayeb, and L. Szczecinski, "Spatial modulation: optimal detection and performance analysis," IEEE Commun. Lett., vol. 12, no. 8, pp. 545-547, Aug. 2008.

[6] J. Jeganathan, A. Ghrayeb, L. Szczecinski, and A. Ceron, "Space shift keying modulation for MIMO channels," IEEE Trans. Wireless Commun., vol. 8, no. 7, pp. 3692-3703, July 2009.

[7] P. Wolniansky, G. Foschini, G. Golden, and R. Valenzuela, "V-BLAST: an architecture for realizing very high data rates over the rich-scattering wireless channel," in Proc. IEEE Int. Symp. Signals, Syst., Electronics, Sep./Oct. 1998, pp. 295-300.

[8] S. M. Alamouti, "A simple transmit diversity technique for wireless communications," IEEE J. Sel. Areas Commun., vol. 16, no. 8, pp. 14511458, Oct. 1998.
[9] L. Xiao, L. Greenstein, N. Mandayam, and W. Trappe, "Using the physical layer for wireless authentication in time-variant channels," IEEE Trans. Wireless Commun., vol. 7, no. 7, pp. 2571-2579, July 2008.

[10] J. Jeganathan, A. Ghrayeb, and L. Szczecinski, "Generalized space shift keying modulation for MIMO channels," in Proc. IEEE Int. Symp. Personal, Indoor, Mobile Radio Commun., Sep. 2008, pp. 1-5.

[11] R. Y. Mesleh, M. Di Renzo, H. Haas, and P. M. Grant, "Trellis coded spatial modulation," IEEE Trans. Wireless Commun., vol. 9, no. 7, pp. 2349-2361, July 2010.

[12] A. Alshamali and B. Quza, "Performance of spatial modulation in correlated and uncorrelated Nakagami fading channel," J. Commun., vol. 4, no. 3, pp. 170-174, Apr. 2009.

[13] M. K. Simon and M.-S. Alouini, Digital Communication over Fading Channels: A Unified Approach to Performance Analysis, 1st edition. John Wiley \& Sons, Inc., 2000.

[14] H. Hashemi, "The indoor radio propagation channel," Proc. IEEE, vol. 81, no. 7, pp. 943-968, July 1993.

[15] M. Di Renzo, F. Graziosi, and F. Santucci, "A unified framework for performance analysis of CSI-assisted cooperative communications over fading channels," IEEE Trans. Commun., vol. 57, no. 9, pp. 2552-2557, Sep. 2009.

[16] M. Di Renzo, F. Graziosi, and F. Santucci, "A comprehensive framework for performance analysis of dual-hop cooperative wireless systems with fixed-gain relays over generalized fading channels," IEEE Trans. Wireless Commun., vol. 8, no. 10, pp. 5060-5074, Oct. 2009.

[17] H. L. Van Trees, Detection, Estimation, and Modulation Theory, Part I: Detection, Estimation, and Linear Modulation Theory. John Wiley \& Sons, Inc. 2001.

[18] M. Abramowitz and I. A. Stegun, Handbook of Mathematical Functions with Formulas, Graphs, and Mathematical Tables, 9th edition. New York: Dover, 1972.

[19] A. P. Prudnikov, Y. A. Brychkov, and O. I. Marichev, Integrals and Series-Vol. 3: More Special Functions. 2003.

[20] D. Tse and P. Viswanath, Fundamentals of Wireless Communication. Cambridge University Press, 2005.

[21] R. A. Amaral de Souza and M. D. Yacoub, "Bivariate Nakagami$m$ distribution with arbitrary correlation and fading parameters," IEEE Trans. Wireless Commun., vol. 7, no. 12, pp. 5227-5232, Dec. 2008.

[22] G. K. Karagiannidis, D. S. Zogas, and S. A. Kotsopoulos, "On the multivariate Nakagami- $m$ distribution with exponential correlation," IEEE Trans. Commun., vol. 51, no. 8, pp. 1240-1244, Aug. 2003.

[23] P. Dharmawansa, N. Rajatheva, and C. Tellambura, "Infinite series representations of the trivariate and quadrivariate Nakagami- $m$ distributions," IEEE Trans. Wireless Commun., vol. 6, no. 12, pp. 4320-4328, Dec. 2007.

[24] M. K. Simon and M.-S. Alouini, "A unified approach to the performance analysis of digital communications over generalized fading channels," Proc. IEEE, vol. 86, no. 9, pp. 1860-1877, Sep. 1998.

[25] A. Annamalai, C. Tellambura, and V. K. Bhargava, "A general method for calculating error probabilities over fading channels," IEEE Trans. Commun., vol. 53, no. 5, pp. 841-852, May 2005.

[26] M. Di Renzo and H. Haas, "Performance comparison of different spatial modulation schemes in correlated fading channels," in Proc. IEEE Int. Conf. Commun., May 2010, pp. 1-6.

[27] V. S. Adamchik and O. I. Marichev, "The algorithm for calculating integrals of hypergeometric type functions and its realization in REDUCE system," in Proc. ACM International Conf. Symbolic Algebraic Computation, Aug. 1990, pp. 212-224.

[28] K. Roach, "Meijer-G function representations," in Proc. ACM International Conf. Symbolic Algebraic Computation, July 1997, pp. 205-211.

[29] S. Haykin, Communication Systems, 4th edition. Wiley, 2000.

[30] J. Reig, M. A. Martinez-Amoraga, and L. Rubio, "Generation of bivariate Nakagami- $m$ fading envelopes with arbitrary not necessary identical fading parameters," Wireless Commun. Mobile Comput., vol. 7, no. 4, pp. 531-537, May 2007.

[31] Q. T. Zhang, "A decomposition technique for efficient generation of correlated Nakagami fading channels," IEEE J. Sel. Areas Commun., vol. 18 , no. 11 , pp. 2385-2392, Nov. 2000.

[32] M. Z. Win, G. Chrisikos, and J. H. Winters, "MRC performance for $M$-ary modulation in arbitrarily correlated Nakagami fading channels," IEEE Commun. Lett., vol. 40, no. 10, pp. 301-303, Oct. 2000.

[33] C. B. Dietrich, K. Dietze, J. R. Nealy, and W. L. Stutzman, "Spatial, polarization, and pattern diversity for wireless handheld terminals," IEEE Trans. Antennas Propagat., vol. 49, no. 9, pp. 1271-1281, Sep. 2001.

[34] M. Di Renzo and H. Haas, "Improving the performance of space shift keying (SSK) modulation via opportunistic power allocation," IEEE Commun. Lett., vol. 14, no. 6, pp. 500-502, June 2010. 
[35] M. Nakagami, "The $m$-distribution: a general formula of intensity distribution of rapid fading," in Statistical Methods in Radio Wave Propagation, W. G. Hoffman, editor. Oxford, U.K.: Permagon Press, 1960, pp. 3-36.

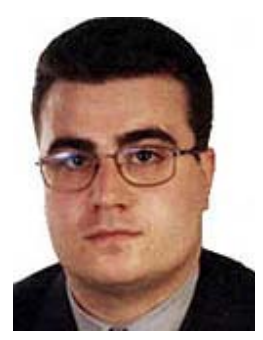

Marco Di Renzo (SM'05-AM'07-M'09) was born in L'Aquila, Italy, in 1978. He received the Laurea (cum laude) and the Ph.D. degrees in electrical and information engineering from the Department of Electrical and Information Engineering, University of L'Aquila, Italy, in April 2003 and January 2007, respectively. From August 2002 to January 2008, he was with the Center of Excellence for Research DEWS, University of L'Aquila, Italy. From February 2008 to April 2009, he was a Research Associate with the Telecommunications Technological Center of Catalonia, Barcelona, Spain. From May 2009 to December 2009, he was a Research Fellow with the Institute for Digital Communications (IDCOM), The University of Edinburgh, Edinburgh, Scotland, United Kingdom (UK). Since January 2010, he has been a Researcher ("Chargé de Recherche") with the French National Center for Scientific Research (CNRS), and a research staff member of the Laboratory of Signals and Systems (LSS), a joint research laboratory of the CNRS, the École Supérieure d'Électricité (SUPÉLEC), and the University of Paris-Sud XI, Paris, France. His main research interests are in the area of wireless communication theory, signal processing, and information theory.

In December 2004, he was a co-founder of WEST Aquila s.r.l. (Wireless Embedded Systems Technologies L'Aquila), an R\&D Spin-Off of the Department of Electrical and Information Engineering, and the Center of Excellence for Research DEWS, where he currently holds the position of Senior Research Engineer in wireless communications. In 2006, he was a Visiting Scholar with the Mobile and Portable Radio Research Group (MPRG), in the Bradley Department of Electrical and Computer Engineering, Virginia Polytechnic Institute and State University, USA.

Dr. Di Renzo was awarded a special mention for the outstanding fiveyear (1997-2003) academic career from the University of L'Aquila, Italy; a three-year Ph.D. fellowship (ranked 1st) from the Department of Electrical and Information Engineering, University of L'Aquila, Italy, and THALES Communications s.p.a, Land and Joint Systems Division of Advanced Studies, Chieti, Italy; and a personal "Torres Quevedo" Grant (PTQ-08-01-06437) from the "Ministry of Science and Innovation" in Spain for his research on ultra wide band systems and cooperative localization for wireless sensor networks.

Dr. Di Renzo is a Member of the IEEE and the IEEE Communications Society, and serves as reviewer for transaction journals and international conferences. He served as Publicity Chair of the 2010 International Conference on Mobile Lightweight Wireless Systems (Mobilight). He also serves as Technical Program Committee (TPC) member and Session Chairman of several international conferences in communications.

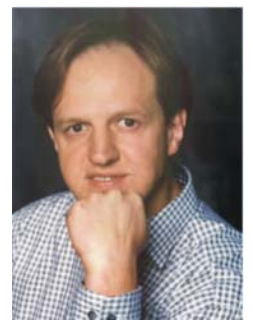

Harald Haas (SM'98-AM'00-M'03) received the $\mathrm{Ph} . \mathrm{D}$. degree from the University of Edinburgh in 2001. His main research interests are in the areas of wireless system engineering and digital signal processing, with a particular focus on interference aware MAC protocols, multiuser access, link adaptation, scheduling, dynamic resource allocation, multiple antenna systems, and optical wireless communication.

He joined the International University Bremen (Germany), now Jacobs University Bremen, in September 2002 where he is now Honorary Professor of electrical engineering. In June 2007, he joined the University of Edinburgh (Scotland/UK) where he is Professor of Mobile Communications in the Institute for Digital Communications (IDCOM).

Haas received a best paper award at the International Symposium on Personal, Indoor and Mobile Radio Communications (PIMRC) in Osaka/Japan in 1999 and holds more than 15 patents in the area of wireless communications. Haas contributed a chapter to the Handbook of Information Security entitled "Air Interface Requirements for Mobile Data Services" by John Wiley \& Sons, Inc. He s co-author of a book entitled Next Generation Mobile Access Technologies: Implementing TDD with Cambridge University Press. His work on optical wireless communication was selected for publication in 100 Produkte der Zukunft (100 Products of the Future) authored by Nobel Laureate T. W. Hänsch. Since 2007, Haas has been a Regular High Level Visiting Scientist supported by the Chinese "111 program" at Beijing University of Posts and Telecommunications. 